\title{
Design and Experimental Investigation of a Novel Spiral Microfluidic Chip to Separate Wide Size Range of Micro-Particles Aimed at Cell Separation
}

\section{Seyed Ali Tabatabaei}

Tarbiat Modares University

Mohammad Zabetian targhi ( $\nabla$ zabetian@modares.ac.ir)

Tarbiat Modares University https://orcid.org/0000-0001-5622-2639

\section{Research}

Keywords: microparticle isolation, spiral microchannel, microfluidic separation, passive separation

Posted Date: January 11th, 2021

DOl: https://doi.org/10.21203/rs.3.rs-142210/v1

License: (c) (1) This work is licensed under a Creative Commons Attribution 4.0 International License.

Read Full License 


\title{
Design and Experimental Investigation of a Novel Spiral Microfluidic Chip to Separate Wide Size Range of Micro-Particles Aimed at Cell Separation
}

\author{
Seyed Ali Tabatabaei ${ }^{1}$, Mohammad Zabetian Targhi ${ }^{2,}{ }^{*}$ \\ ${ }^{1}$ Department of Mechanical Engineering, Tarbiat Modares University, Tehran, Iran, alitabatabaei@modares.ac.ir \\ ${ }^{2}$ Department of Mechanical Engineering, Tarbiat Modares University, Tehran, Iran, zabetian@ modares.ac.ir
}

\begin{abstract}
Background

Isolation of microparticles and biological cells on microfluidic chips has received considerable attention due to their applications in numerous areas such as medical and engineering fields. Microparticles separation are of great importance in bioassays owing to the need for a smaller sample and device size, and lower manufacturing costs.

In this study, we first explain the concepts of separation and microfluidic science along with their applications in the medical sciences, and then, a conceptual design of a novel inertial microfluidic system is proposed and analyzed. The PDMS spiral microfluidic device was fabricated, and its effects on the separation of particles with sizes similar to biological particles were experimentally analyzed. This separation technique can be used in the process of separating cancer cells from the normal ones in the blood samples.
\end{abstract}

\section{Results}

These components required for testing were selected, assembled, and finally, a very affordable microfluidic kit was provided. Different experiments were designed, and the results were analyzed using appropriate software and methods. Separator system tests with polydisperse hollow glass particles (diameter 2-20 $\mu \mathrm{m}$ ), and monodisperse Polystyrene particles (diameter 5,15 $\mu \mathrm{m}$ ), and the results exhibit an acceptable chip performance with 86 percent of efficiency for both monodisperse particles and polydisperse particles. The microchannel collects particles with an average diameter of $15.8 \mu \mathrm{m}, 9.4 \mu \mathrm{m}$, and $5.9 \mu \mathrm{m}$ at the Proposed reservoirs.

\section{Conclusion}

This chip can be integrated into a more extensive point-of-care diagnostic system to test blood samples, and it could be said Based on the results of the experiments, this low-cost and user-friendly setting can be used for a variety of microparticle separation programs such as cell separation in biological assays.

Keywords: microparticle isolation, spiral microchannel, microfluidic separation, passive separation 


\section{Nomenclature}

$\begin{array}{lll}C_{L}: \text { Lift coefficient } & F_{D}: \text { Drag force } & R_{c}: \text { Radius of curvature } \\ d: \text { particle diameter } & F_{D, \text { Dean }}: \text { Dean drag force } & \operatorname{Re}: \text { Reynolds number } \\ D_{h}: \text { Hydraulic diameter } & F_{m}: \text { Magnetic force } & G \text { : Shear rate of flow } \\ D e: \text { Dean number } & U: \text { Velocity of fluid flow } & h: \text { Channel height } \\ F_{L}: \text { Lift force } & U_{\text {max }}: \text { Maximum fluid velocity } & \rho: \text { Density } \\ F_{W L}: \text { Wall-induced lift force } & U_{F}: \text { Average flow velocity } & \mu: \text { Viscosity of fluid } \\ F_{S L}: \text { Shear-gradient lift force } & U_{\text {Dean }}: \text { Dean velocity } & \chi: \text { dimensionless distance for particles }\end{array}$

\section{Background}

Isolation of bio-components is of particular importance not only for the identification and study of bio-particles for detection, improvement, or treatment of diseases but also the improvement of health status. Separation of Biological elements can also have a specific role in the sampling process, studying the impact of treatment, medical diagnosis, and the desired tests. Separation of microparticles can be used for study and research in cases such as bio sample preparation steps in the molecular analysis, including chemical and biological analyses such as food and chemical processing and also environmental assessment automotive and electronics industries [1]-[7]. Moreover, the separation of biological elements is also used at different stages of understanding diseases, treatment processes, and medications. Bio-particle focusing applications in clinical diagnostics, Efficient separation of human T-lymphocytes (CD4+) from whole blood as a step in the diagnosis and treatment of HIV disease, separation of neuroblastoma and glioma cells in cell replacement therapy of neurodegenerative disorders, Blood cell separation in blood analysis for both diagnostics and therapeutics, airway secretion cell isolation for pulmonary diseases, and applications such as preparation of biological samples are just some of the applications of particle separation in the medical field [8]-[12]. Isolation of circulating cancer cells from blood samples is One of the most critical applications of bio-particle isolation.

Nowadays, cancer is one of the most important causes of death around the world. It is estimated that The number of deaths from cancer rises to 13 million in 2030. Nevertheless, according to the World Health Organization (WHO), it can be that about $30 \%$ of cancer-related deaths would be avoidable if, before the occurrence of cancer metastases, patients would be diagnosed and treated, since metastases are said to cause $\sim 90 \%$ of cancer-related deaths.[13]. Cancer metastases occur when Circulating tumor cells (CTCs) flow into the bloodstream from the main tumor sites. CTCs are cancer cells that are circulating in the blood shed from primary tumors and flow through the bloodstream to other parts of the body. Moreover, CTCs isolation and analysis are also essential for investigation of cancer[14]. The presence and frequency of CTCs in bloodstreams of cancer patients are essential to primal cancer detection and treatment monitoring. Furthermore, an important approach to analyze CTCs is through isolation and enrichment of a large number of those cells, which is pivotal to elucidating the mechanisms underlying the metastatic process. isolated CTCs can be used in cell and molecular analysis, especially for personalized cancer therapies. Additionally, the enrichment and viable CTCs culture from blood of cancer patients will create many opportunities for the development 
of new anti-cancer drugs for Future therapeutic goals[15]. Isolation and cell counting are also required to monitor post-treatment in many types of cancer therapy[16].

Nowadays, with the advancement of microfluidics science, the number of studies on the isolation of bio-particles on microfluidic chips has increased, this will result in a comprehensive examination of the common diseases in the world in the medical and engineering fields. Previous micro-particle separation methods such as FACS ${ }^{1} \&$ MACS $^{2}$ are expensive, requiring a trained technician for operation and maintenance, and their complex setup and operation for active sorting requires complicated fabrication and several steps which make it a time-consuming process and lead to cell loss [17]-[21]. These systems require costly instrumentation, skilled operators, and multiple passes for full analysis[20]. One of the main setbacks of conventional methods is due to their lack of susceptibility to the viability of isolated cells[22].

Due to the weaknesses of conventional methods, there has been a profound interest in using techniques that take advantage of microscale technologies and the inherent properties of cells for improved mechanization and reduced cost[2]. Microfluidic talks about the science that arranges with the behavior, precise control, and manipulation of fluids and particles in the scale of tens to hundreds of micrometers. With the appropriate length scale that matches the scales of cells, microfluidics is well suited to contribute significantly to cell biology. This scale provides an interface for manipulating single cells and accessing these forces in a variety of ways, including for kinetic, equilibrium, and elution separation. They will also possibly reduce sample volume and cost and are potentially portable[23]. Therefore, as an important step in many biological and medical analysis, the need for efficient cell separation has led to the recent development of numerous microfluidics separation techniques. Microfluidics has the potential to reduce the limitations of previous methods of polling and testing on samples. The application of microfluidics-based technologies for cell separation offering numerous advantages including reduced sample volumes; reduced sample preparation procedures and leading to faster sample processing and reducing analysis time; high sensitivity and spatial resolution; increasing detection accuracy; reducing odds of sample contamination; The potential for the designs that are sufficiently compact for integration into point-of-care systems for clinical diagnostics, and the potential to be highly integrated and automated to reduce human intervention and errors as well as device errors[24]-[28]. Microfluidics also presents a functional tool-set for cell separation that offers better control of the microenvironment during separation and facilitates integration and automation for high throughput sample processing [23]. Therefore, the development of efficient microscopic separation methods can provide greater control over cell size distribution is increasing the importance of the realization of many on-chip laboratory systems.

Mechanical and physical properties, including size, shape, density, adhesion, and deformability, are common markers for differentiation. In microfluidic separation science, separation methods are classified as active, passive, and combined methods. The active separation methods use external forces to exert force. Magnetic, dielectrophoresis, and acoustophoresis separation techniques are examples of active methods that separate particles by applying external force[29]-[33]. In the passive separation method, in contrast to the active methods, external force fields are not used,

\footnotetext{
${ }^{1}$ Fluorescence Activated Cell Sorting

2 Magnetic Activated Cell Sorting
} 
and therefore, using properties such as channel geometry and inherent hydrodynamic forces is significant. In passive methods, techniques such as inertia, pinch flow fraction, deterministic lateral displacement, filtration, etc. are used for separation[34]-[39]. Depending on how it works, the basis of passive separation is either the difference in particle size or density. Moreover, since passive isolation techniques do not use external force, these techniques are simpler to use in comparison to the active techniques, and by using passive techniques for separation, cell viability will significantly decrease. In microfluidics, passive cell separation techniques have been extensively studied. This is due to the fact that these techniques utilize simple channel geometries and pressurized flows to achieve and enhance separation. There has always been a trade-off between separation efficiency and operating power[10], and this has led the researchers to the combined methods. In the combined separation method, active and passive separation methods are used simultaneously, thus improving separation and sorting performance.

Due to the simplicity of passive separators and since it causes less damage to cells in this method, the development of inertial separation techniques, one of the passive methods, has been the focus of recent years. Inertial microfluidic techniques work in the intermediate Reynolds number range $(\sim 1<\operatorname{Re}<\sim 100)$ between Stokes and turbulent regimes. In this intermediate range, both inertia and fluid viscosity are finite and bring about several intriguing effects that form the basis of inertial microfluidics. On the other hand, according to Reynolds number of flow, inertial microfluidic systems are expected to have a critical impact on high-throughput separation applications in fluids processing such as particle separation[4],[17]. The inertial displacement was first expressed by Segre and Silberberg [41] in the 1960s. This migration behavior takes advantage of hydrodynamic forces that act on particles, localize them within the flow caused by the balance of lift forces arising from the parabolic velocity profile (the shear-induced inertial lift), and the interaction between particles and the channel wall (the wall-induced lift)[10].In recent years, extensive researches have been done on inertial separation technique, and inertial devices can be divided into three general categories: straight channels, straight channels with pillar arrays or multi orifice structures, and spiral channels[24]. In general, a straight channel has the advantage of simplicity and ease of operation. However, this type of channel is usually long, increasing flow resistance and lead to a large machine footprint. Creating a secondary flow by channel curvature or orifice structure will not only help improve inertial migration but also alter the final equilibrium positions. Due to this reason, the design and use of curved microchannels have received considerable attention. Recently, many investigations have been done on curved microchannels in the fields of different geometries, different cross-sections, and different applications for separating particles of diverse diameter with appropriate separation rates and efficiency [38]-[42]. It has also been used in some cases to increase the separation and efficiency rates of multi-channel systems in series or parallel with each other[47], [48]. However, passive microfluidics methods have an inherent limitation, in which it is difficult to separate cells of the same size and researchers have to adopt a sorting strategy which leads to a trade-off either sacrificing purity for high recovery rate or sacrificing recovery for high purity[49]-[52].

Therefore, In the present work, we have attempted to design a system for microparticle separation to overcome the limitations of the inertial method using a combination of inertial and magnetic techniques. We have used the designed spiral microchannel as an inertial separator to isolate both monodisperse and polydisperse particles with different sizes. The spiral channels can be produced at a meager cost and with a high resolution utilizing PDMS casting; they can also be operated using a double syringe pump, facilitating automation. 


\section{Physics and Equations}

In order to design a separation system, it is necessary to examine the physics of the problem and the forces involved in the flow.

\section{Inertia isolation}

Although the phenomenon of inertial migration has been studied for the past 40 years, its applications have been useful recently, because it has recently been recognized that the effects of inertia and order are beneficial in microfluidics and in cases where their use may have a profound effect. The inertial separation technique utilizes the lift force to balance the particles in distinct transverse positions at the microchannel cross-section, which owes to different transverse positions due to the ratio of particle size to microchannel dimensions. Floating particles flow in the microchannel under the influence of lift forces, migrating to different positions along with the channel range. The key parameters in controlling the magnitude and orientation of lift forces and particle positionings, such as channel dimensions, aspect ratios, particle diameter and flow velocity[8], can be discussed with connection to the relevant equations.

For a particle, located in a direct wall-bounded Poiseuille flow, in addition to a viscous drag force along the axis (according to Eq.1), there are four lateral forces in the particle that operate: Magnus force due to slip- rotation, Safman force due to slip shear, the shear force due to disturbance in the flow field around particles from the wall, and shear gradient lift force due to the curvature of the undisturbed fluid velocity profile (Poiseuille profile). Among these four lift forces, the Magnus force and the Safman force in microfluidics problems are often minimal and negligible[11]. Thus, particle focusing along channel cross-section is dependent on the balance of the shear-induced lift force (arising from the parabolic velocity profile) and the wall-induced lift force (arising from interactions with channel walls), based on their size relative to the microchannel dimensions, thus achieving separation[53]:

$$
F_{D}=6 \pi \mu d U
$$

Where $d$ is particle diameter, $\mu$ is the viscosity of the fluid, and $U$ is the velocity of fluid flow. In the case of the effect of wall-induce lift force for a particle moving near the channel wall, the interaction between the particle and the wall will stop the particle from tracking the fluid flow. Besides, the shrinkage of flow between the particle and the channel wall causes the fluid flow to accelerate to the top of the particle as more flow streamlines diverge. This creates less relative pressure than the "sidewall" of the particles and lifts force away from the wall (Eq.2). In the case of sheargradient lift force, due to the parabolic velocity profile of the in-channel flow, the velocity magnitude varies on each side of the particle, resulting in a pressure difference between the upper and lower sides of the particle. Due to the pressure difference, a shear-gradient lift force is applied to the particles that drive toward the channel wall(Eq.3) [25].

$$
\begin{gathered}
F_{W L}=\frac{\rho U_{m}^{2} d^{6}}{D_{h}^{4}} f_{W L}\left(\operatorname{Re}_{c}, x\right) \\
F_{S L}=\frac{\rho U_{m}^{2} d^{3}}{D_{h}} f_{s L}\left(\operatorname{Re}_{c}, x\right)
\end{gathered}
$$


Where $D_{h}$ is the hydraulic diameter of the channel and $f\left(R_{c}, x\right)$ is the lift coefficient, which depends on the particle position within the channel $(x)$ and the channel Reynolds number $\left(\operatorname{Re}_{c}\right)$. As a result of these two opposing lifting forces, the particles are balanced at predetermined positions around the microchannel margin. This effect is leading the particles with a size comparable to microchannel dimensions' $d / h \geq 0.07$. The magnitude of $F_{L}$ is given by Eq.4:

$$
F_{L}=C_{L} \rho G^{2} d^{4}
$$

where $C_{L}$ is the lift coefficient, which is a function of the particle position across the channel cross-section while assuming an average value of 0.5 , and $G$ is the shear rate of the fluid. The average value of $G$ for a Poiseuille flow is assumed by $G=U_{\max } / D_{h}$, where $U_{\max }$ is the maximum fluid velocity and can be estimated as $2 \times U_{F}$.

$$
F_{L}=\frac{2 \rho U_{F}^{2} d^{4}}{D_{h}^{2}}
$$
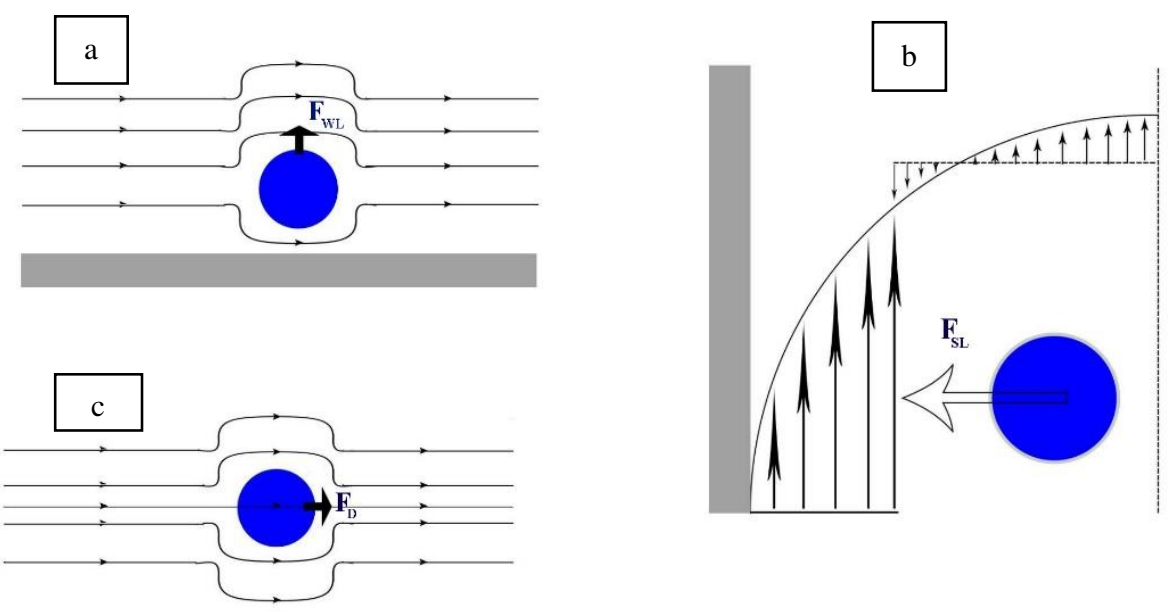

Figure 1: a: Wall-induced lift force that causes the particle to move away from the walls due to the effect of the wall and to approach the center of the channel, b: Shear-induced lift force caused by the parabolic flow velocity profile of the fluid and directs the particle to the sides of the channel, c: Viscous drag force along the axis of flow [25]

As depicted in Fig.2. The focus of particle equilibrium positions due to inertia lift depends on the symmetry of the channel. The particles in the cylindrical channels are cyclically concentrated between the center and wall of the channel according to the type of channel symmetry. According to the interaction between two lift forces, the flow of particles flowing through the square channels is also focused on channel symmetry in four positions to concentrate on the equilibrium positions leads to defined equilibrium positions in channel flows. Interestingly, as the ratio of the channel dimensions becomes larger and becomes a rectangular channel in a very wide or very long channel, symmetry is followed, and mainly two equilibrium positions in the long channel are reduced[8]. 


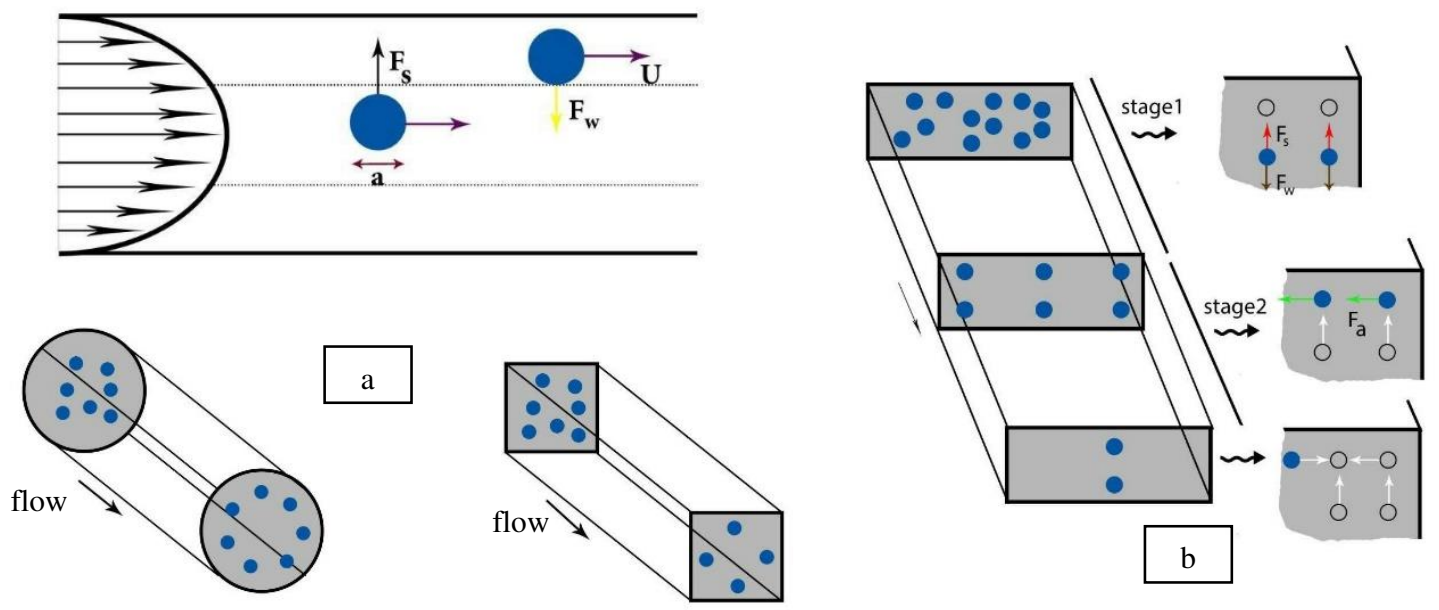

Figure 2: Equilibrium positions of particles in microchannels with different cross-sectional area indicate that a: in circular and square microchannels, the number of positions is high and, b: by reducing the aspect ratios, it is possible to reduce the interchangeable positions similar to rectangular cross-section[10]

\section{Physics of secondary flow and application}

As stated, the inertia separation technique utilizes the lift force to balance the particles in distinct lateral positions at the microchannel cross-section, which is due to the ratio of particles size to microchannel dimensions. However, the micro channel's shape can be used to apply the flow properties as inertia separators. Despite the development and use of straight microchannels for particle sorting by inertia, some challenges still remain. In this type of microchannels, multiple lateral positions make it difficult to extract more than two different types of particles. In order to sort the different particles with high purity and efficiency, it is desirable to reduce the number of focus positions for each particle to one so that a planar output scheme can extract the particles easily. In addition, the long length of straight microchannels has challenged their construction. Implementing devices as low-dimensional channels offer more straightforward construction and ultimately improves efficiency and reduces device cost[10]. The secondary flow on the cross-sectional plane of the channel, which was first reported by William Dean in 1928 [54], occurs in a curved microchannel and happens because the fluid pressure at the inner wall is slightly higher than that of the outer wall. Therefore, the fluid from the center of the channel is pushed outer as a result of this pressure gradient, and the fluid near the top and bottom walls moves to the inner side, satisfying the conservation of mass[3].

The use of curved channels will create a secondary flow due to the centrifugal effect called the flow of Dean. Furthermore, as shown in Fig.3, In curved microchannels, the viscous drag force consists of two components, the mainstream direction due to the axial velocity difference between the fluid and the suspended particles, and the crosssection direction, due to the secondary flow induced by the channel curvature[11]. These pressure and velocity gradient imbalance caused by Dean instability results in secondary flow defined by a non-dimensional number called the Dean number that can be calculated by Eq.7 [55]: 


$$
D e=\frac{\rho U_{F} D_{h}}{\mu} \sqrt{\frac{D_{h}}{2 R_{c}}}=\operatorname{Re} \sqrt{\frac{D_{h}}{2 R_{c}}}
$$

where $\rho$ is the fluid density, $U_{F}$ is the average flow velocity, $\mu$ is the viscosity of the fluid, $R_{C}$ is the radius of curvature of the path of the channel, $D_{h}$ is the channel hydraulic diameter, and Re is the flow Reynolds number. the magnitude of Dean drag force is also given by Stokes' law:

$$
F_{D, \text { Dean }}=3 \pi \mu U_{\text {Dean }} d
$$

In equation (8), $U_{\text {Dean }}$ is the velocity depending on the Dean number, and can be calculated as follows:

$$
U_{\text {Dean }}=1.8 \times 10^{-4} D e^{1.63}
$$

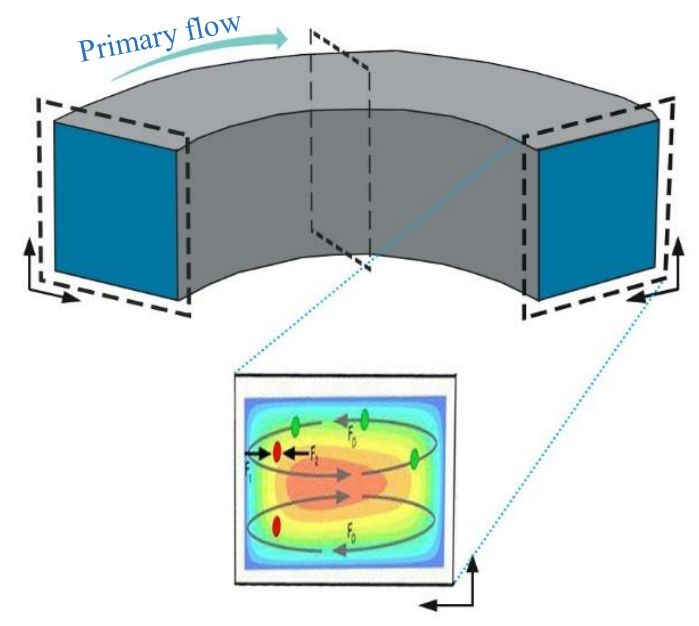

Figure 3: The Dean flow. In curved channels, when inertia is of the highest importance, the faster movement of liquids near the center of the channel tends to continue externally, and in order to maintain mass, more stagnant fluid circulates inwardly near the walls. These two anti-rotation vortices create a perpendicular motion to the mainstream direction[8].

According to Equations 7-9, The parameters that influence the secondary flow and consequently, Dean force in a curved channel are Dean number, Reynolds number, and aspect ratio of the channel[11]. Subsequently, the magnitude of $F_{D}$ can be equal to, larger, or smaller than $F_{L}$ depending on the flow rate and geometry. At meager flow rates, when $\mathrm{Re}$ is low, all the particles occupy equilibrium positions at the center of the channel, as the Re increases, drag force caused by Dean Vortices becomes gradually stronger than the centrifugal force, and the particles tend to move inward of the channel radially. As a result, small particles move closer to the inner wall, while bigger particles remain at their original equilibrium positions.

In this case, the concentration of the particles in different lateral positions depends on the ratio of the lift force discussed earlier, and the dean drag force created by dean flow. As a result, it will require a shorter distance to focus the current, Providing reduced hydrodynamic resistance, which is promising for focusing or separating inertia[56]. The spiral microchannels are appropriate to concentrate larger target particles near the inner wall while smaller particles scatter and move along the flow lines. As a result, different focusing positions can be obtained at the output 
for different sized particles in the channel. Therefore, larger particles are concentrated and aligned near the inner wall, while smaller particles are located near the outer wall. Apart from the dean drag force, larger particles of comparable diameters to the microchannel also experience considerable inertia ( $\mathrm{Re}$ ) forces (both shear and wall-induced), resulting in their concentration and equilibrium. The inertia lift force is effective for larger particles of size comparable to the micro-channel dimensions ( $d / h \geq 0.07$ where $d$ is the particle diameter and $h$ is the channel height) [43]. Whereas the effect of secondary flow on smaller particles is noticeable and contributes to their concentration on the outer wall. Therefore, the device height, particle sizes, and outlet positions can be designed to match a single outlet to each stream containing target-bound beads of a single size, which can thus be separated (Fig.4).

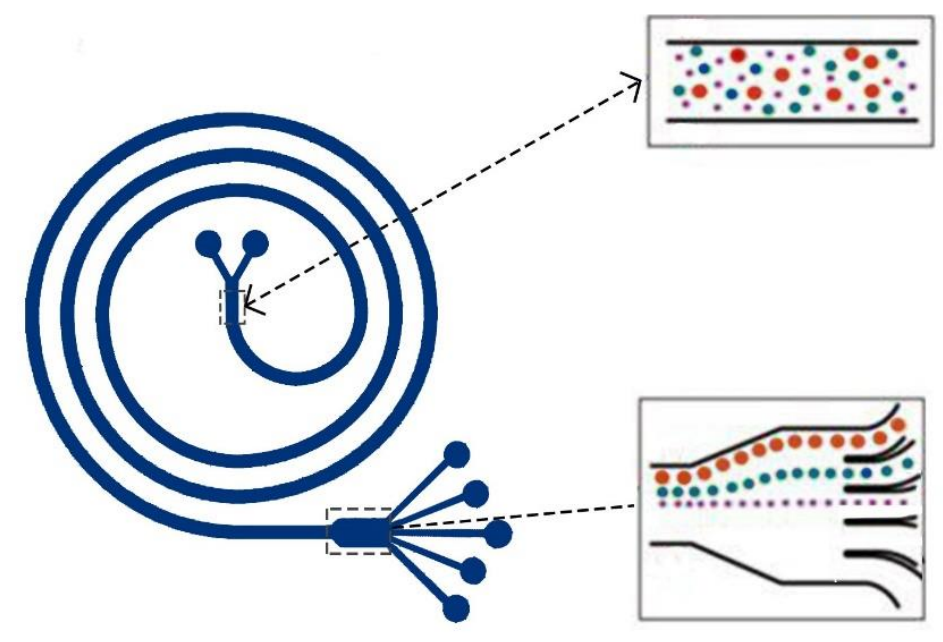

Figure 4: Schematic of the spiral microparticle separator. The randomly dispersed particles equilibrate at different equilibrium positions along the inner wall (IW) of the spiral microchannel under the influence of $F_{L}$ and $F_{D}$. The separation between individual particle streams is enhanced by connecting the spiral section into a diffuser section, before conducting the individual streams using multiple outlet ports[9].

\section{Design principles}

As it was mentioned, it can be seen that by means of a spiral microchannel, the particles in the bloodstream can be separated according to their difference in size and density. In general, the essential microparticles in the blood sample can be divided into three groups depending on their size: red blood cells, white blood cells, and circulating tumor cells. However, since some white blood cells are about the same size and density as circulating tumor cells, passive separation techniques are incapable of separating these kinds of cells. Therefore, using magnetic separation in addition to the inertial technique, as a combined method, will likely increase the purity of the isolated cell. Therefore, in the present chip design, it is attempted to use a combined method of magnetic separation along with inertial separation to take advantage of both active and passive methods.

\section{Conceptual Design}

In magnetic separation, the sample cells are first labeled with magnetic beads by antibodies. Then, a magnetic field gradient is used to separate cells with magnetic beads. During the sorting of magnetic cells by crossing the microchannel, cells that get attached to the magnetic beads are deflected in one direction and follow the path of the 
magnetic lines, whereas cells that are not labeled follow the flow direction[1]. Magnetic separation techniques can have several advantages, such as a high percentage of purification and shorter sorting time. It is also cheaper to use a magnetic separation technique than other active methods and is also easier to construct. This method is usually used to distinguish two types of target cells, and it also can be said that it depends on just one single parameter[3]. As shown in Fig.5, the fluid containing the particles enters from one side and the buffer fluid on the other, and then by magnetic field effect on the labeled particles, these particles are diverted and separated.

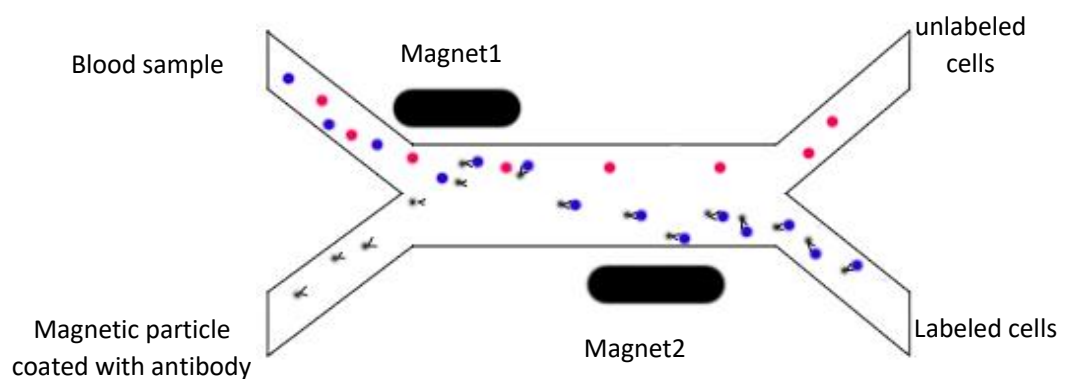

Figure 5: In magnetic microfluidic separation, the arbitrary cells are labeled by antibodies and are pulled out of the flow path via applied magnetic force.

The magnitude of the magnetic force applied to the floating particles in the flow can be calculated by [Eq.10] [57]:

$$
F_{m}=2 \pi \mu_{0} \mu_{f} R_{p}^{3}\left(\frac{\mu_{p}-\mu_{f}}{\mu_{p}+2 \mu_{f}}\right) \nabla H^{2}
$$

whereas $\mu$ is the magnetic permeability, $\mu_{0}$ is the permeability of free space, $R_{p}$ is the radius of particles and $H$ is the amount of magnetic field.

\section{Detailed design}

The designed chip uses inertial isolation technique for size-based separation of tumor cells and WBCs that are equal in size to CTCs from the whole blood, and then uses microfluidic magnetophoresis for isolation of circulating tumor cells. It is currently composed of two separate chips, used serially (in-line). Therefore, this chip can be designed for negative enrichment, where the target cell was unlabeled, and all other cells were labeled with one or more antibodies. As the sample passed through this chip in a flow-through manner, other cells were captured. One of the advantages of negative selection is that a label is not needed for the target cell. Negative enrichment will continue to be an area of interest for CTC collection, as the unlabeled CTCs can readily be used in downstream analyses[58]. This Negative selection strategy provides tumor antigen-independent enrichment performance, and as a result, it applies to cells disseminated from virtually any tumor type[47].

In the designed separation system, as shown in Fig.6, initially, red blood cells and primary white blood cells with a size smaller than the cancer cells were separated by a spiral microchannel. Then, the desired output from the microchannel, which contains circulating Tumor cells and some white blood cells that are equal in size to CTCs, enters the $2^{\text {nd }}$ chip and is combined with magnetic beads. Given that at this stage, there are only two types of cells (circulating 
cancer and white blood cell), isolation can be done by negative selection. In this way, the magnetic beads are labeled on white blood cells, which enables active separation in a designed system, which is not only cheaper and more efficient but also independent from antigens and can be used for a variety of circulating cancer cells. Each chip has a different manufacturing procedure, and these two chips can be considered separately. In the present work, we will perform the relevant experiments for the first chip.

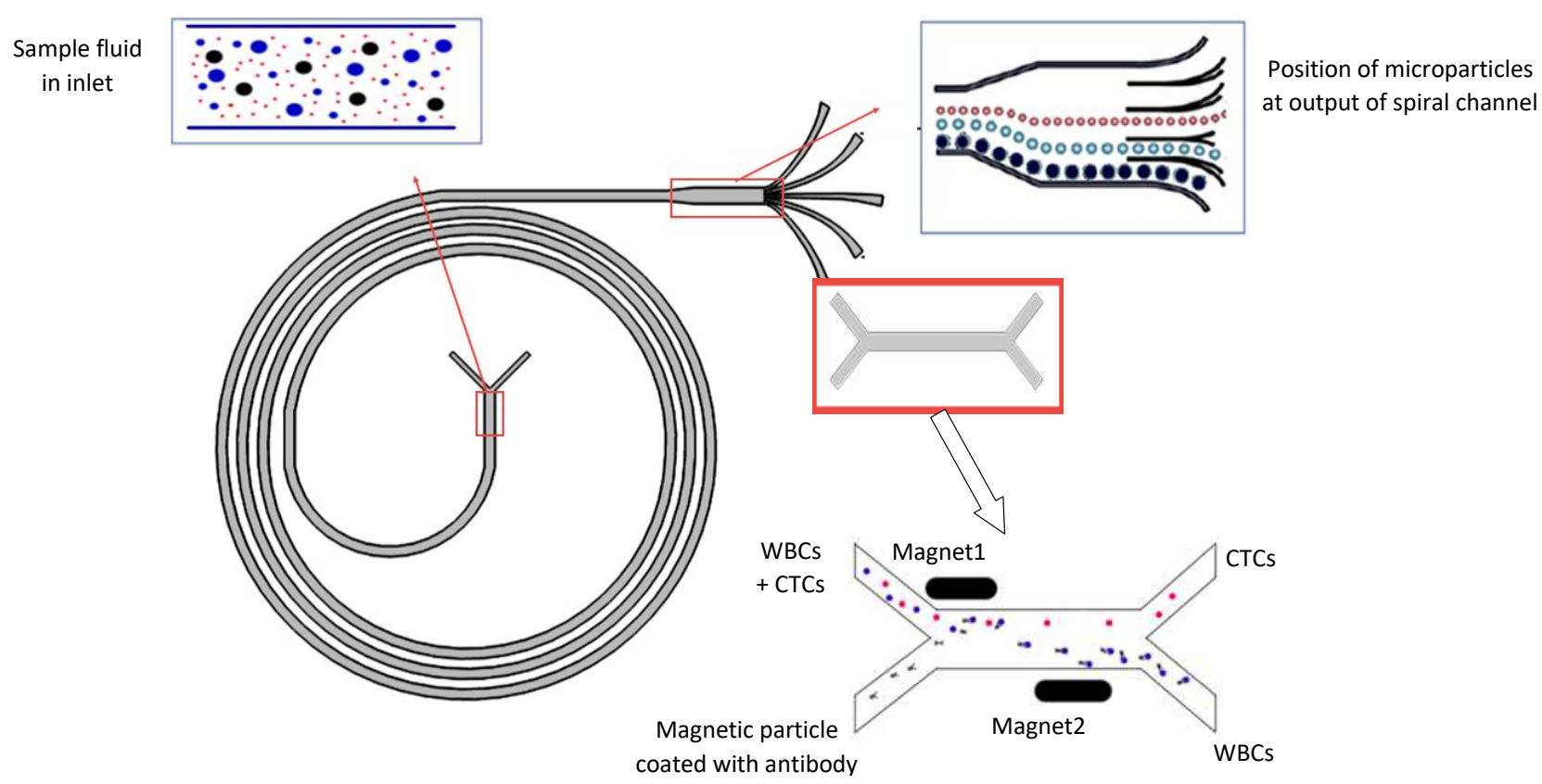

Figure 6: Schematic of the designed separator system: in the first part of the microchannel, the particles are separated by inertia and size-based technique. Both cancer cells and magnetic-labeled particles have been removed. In the magnetic separation section of the labeled white blood cells, they deflect from the current path-line, and as a result, the circulating cancer cells will be selected negatively.

Previously, we talked about the effects of the wall and the wall force, which is applied to particles inside the microchannel. Among the common geometries for the channel cross-sections, the circular and square cross-sections, due to their regularity, cause the wall effect to be uniform in all directions. In this case, the separation of particles in the cross-section due to exit particles of the same size in multiple transverse and longitudinal positions will be virtually impossible. In a rectangular channel, the difference between velocity profiles on the longitudinal and transverse walls of different sizes reduces the particle concentration positions and thus makes it possible to collect particles at the output. As mentioned earlier, in a curved or spiral microchannel, the curved geometry gives rise to the dean force, which is the result of the secondary flow in the cross-section of the channel, the presence of the dean force, disturbing the equilibrium of the two upper forces. The particles are guided by the secondary flow and the effect of the dean force, from the upper and lower walls to the inner and outer walls (in the transverse section). Thus, in the spiral microchannel with the rectangular cross-section, resulting in a possible balance of force for focusing, the same particles become in a single position [59]. Regarding the irregular cross-sections, such as the microchannels with the 
trapezoidal cross-section, due to the secondary flow intensification, can be suitable for separating particles according to their size [45], [56]. However, these channels are suitable for separating just two kinds of particles with different size [42]. Furthermore, size-based enrichment techniques are often unable to recover smaller CTCs[43], [47].

Therefore, in the designed microchannel, a rectangular cross-section is considered in order to take advantage of the desired effects of geometry on the microchannel cross-section. To avoid entrainment of particles in the dean vortices, the dimension of the microchannel should be $d / \mathrm{D}_{\mathrm{h}}<0.07$ [3]. Therefore, the larger side of the rectangular section of the microchannel (microchannel width) is $500 \mu \mathrm{m}$, and the smaller side of the microchannel cross-section (microchannel depth) is considered $130 \mu \mathrm{m}$ in order to better influence the particles. For construction considerations, the depth of all microchannel sections is $130 \mu \mathrm{m}$. In the passive separation part of the system, the spiral microchannel consists of 4 loops, the first loop is $2 \mathrm{~cm}$ in diameter, and the distance between the spirals is $500 \mu \mathrm{m}$. In the microchannel manifold, two inlet plugs are provided for the sample fluid and buffer fluid, with a width of $250 \mu \mathrm{m}$, which will create an angle of 90 degrees between the two plugs. In the microchannel output section, a $500-\mu \mathrm{m}$ microchannel width is pre-outputted to an $800-\mu \mathrm{m}$ transverse section to achieve better separation using layer flow profiles at the outlet that open to 5 outputs of equal width at the termination it ends. Besides, the opening at the microchannel termination reduces the velocity near the output that can allow better imaging of the particles. Due to the manufacturing constraints in the microchannel dimension, to allow more accessible construction of the microchannel, the distance of both consecutive outputs is assumed to be $40 \mu \mathrm{m}$, and the width of each output is 128 $\mu \mathrm{m}$.

The magnetic part of the separation system, which is a direct microchannel, is considered to be consists of two inputs and two outputs. The cross-section of the magnetic section is a rectangle measuring $280 \mu \mathrm{m}$ and $130 \mu \mathrm{m}$ with a depth of $130 \mu \mathrm{m}$. The length of the direct microchannel is $20 \mathrm{~mm}$. At the magnetic section inlet, the fluid containing the particles will be taken from the output of the first section of the system, the symmetric geometry of the buffer fluid entering the microchannel. Within 1 millimeter of the direct microchannel, the permanent magnet is positioned, causing the magnetic field to deviate the labeled particles with magnetic beads, and then, the separated particles will exit the two different outlets.

\section{Results \& Discussion}

As mentioned, the fabricated devices consist of a four-loop spiral microchannel with two inlets and five similarly spaced outlets. The spiral designs have an initial diameter of curvature of $2 \mathrm{~cm}$, with spacing between the successive spiral loops fixed at $500 \mu \mathrm{m}$. The microchannel's width was fixed at $500 \mu \mathrm{m}$, and the microchannel height is $130 \mu \mathrm{m}$. There are two inputs at the beginning, one for the fluid containing particles and the other for the buffer fluid, which is used as a sheath flow to focus small particles. At the outlet, the $500 \mu \mathrm{m}$ width channel is opened into an $800 \mu \mathrm{m}$ wide section to increase the spacing between particle streams before splitting into five $128 \mu \mathrm{m}$ width outlets.

In this work, we have tried to take advantage of differences in particle sizes so that with the difference in the ratio of $F_{L}$ and $F_{D}$ in the spiral channel to separate monodisperse and polydisperse microparticles. In the first test, 
polydisperse HGS particles of 2 to $20 \mu \mathrm{m}$ diameter with an average diameter of $10 \mu \mathrm{m}$ were used. The sheath flow rate is $900 \mu \mathrm{L} / \mathrm{min}$, and the flow rate of Particle Suspension is $300 \mu \mathrm{L} / \mathrm{min}$. Fig. 7 and Table 1 give details of the results of this test. By imaging the reservoirs embedded in the outputs, it can be derived that for large particles, the lift force ongoing to dominate over the Dean force, and obviously, the particles would be focused on the inner wall of the channel. As a result, larger particles in size have been gathered in the first outlet. On the other hand, it is evident from Fig.7-c and Table1 that in the spiral microchannel, for small particles, Dean drag forces are always dominated over the lift forces, which means that these particles are likely to enter the Dean's vortex and circulate along the fluid stream. Accordingly, smaller particles in size are focused closer to the centerline of the microchannel and will exit from the third output.
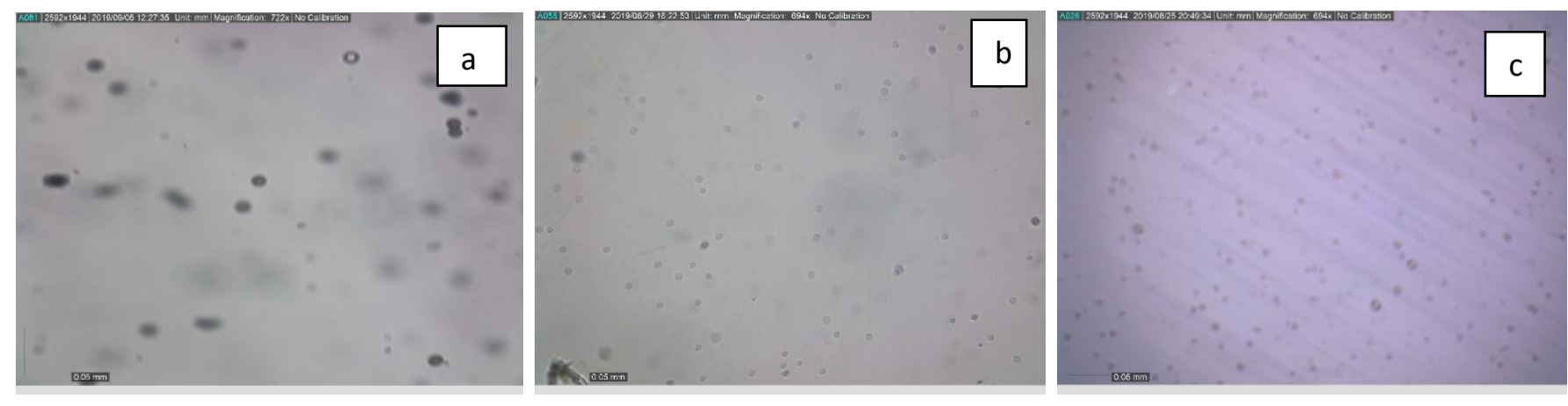

Figure 7: Experiment for polydisperse particles with mean diameter of $10 \mu \mathrm{m}$, sheath flow rate of $900 \mu \mathrm{L} / \mathrm{min}$ and particle suspension flow rate of $300 \mu \mathrm{L} / \mathrm{min}$, a: particles in outlet reservoir No.1, b: particles in outlet reservoir No.2 and c: particles in outlet reservoir No.3

Table 1: average particle diameter at different outlets in Experiment 1

\begin{tabular}{|cccc|}
\hline $\begin{array}{c}\text { Sheath } \\
\text { flow rate }\end{array}$ & $\begin{array}{c}\text { Particle suspension } \\
\text { flow rate }\end{array}$ & Outlet & $\begin{array}{c}\text { Particles'average } \\
\text { diameter }\end{array}$ \\
\hline & & Outlet 1 & $16.96 \pm 0.02 \mu \mathrm{m}$ \\
$900 \pm 1 \mu \mathrm{L} / \mathrm{min}$ & $300 \pm 1 \mu \mathrm{L} / \mathrm{min}$ & Outlet 2 & $8.8 \pm 0.02 \mu \mathrm{m}$ \\
& & Outlet 3 & $5.8 \pm 0.02 \mu \mathrm{m}$ \\
\hline
\end{tabular}

For further studies, similarly, polydisperse HGS particles of 2 to $20 \mu \mathrm{m}$ diameter with an average diameter of 10 $\mu \mathrm{m}$ were used, the flow rate of Sheath flow is $1200 \mu \mathrm{L} / \mathrm{min}$, and the flow rate of Particle Suspension is $300 \mu \mathrm{L} / \mathrm{min}$. As shown in Fig. 8.a, it is clear that also in this test, larger particles in size have been collected in the first outlet. Table 2 shows the results of test 2, the average diameter of particles that have been ejected from the outlet 1 is $15.8 \mu \mathrm{m}$ whereas the average diameter of particles that have been ejected from the outlet 2 is $9.4 \mu \mathrm{m}$. In the third outlet, the average diameter of output Particles is equal to $5.9 \mu \mathrm{m}$, which indicates that Particles of different sizes are collected from the desired outlets. 

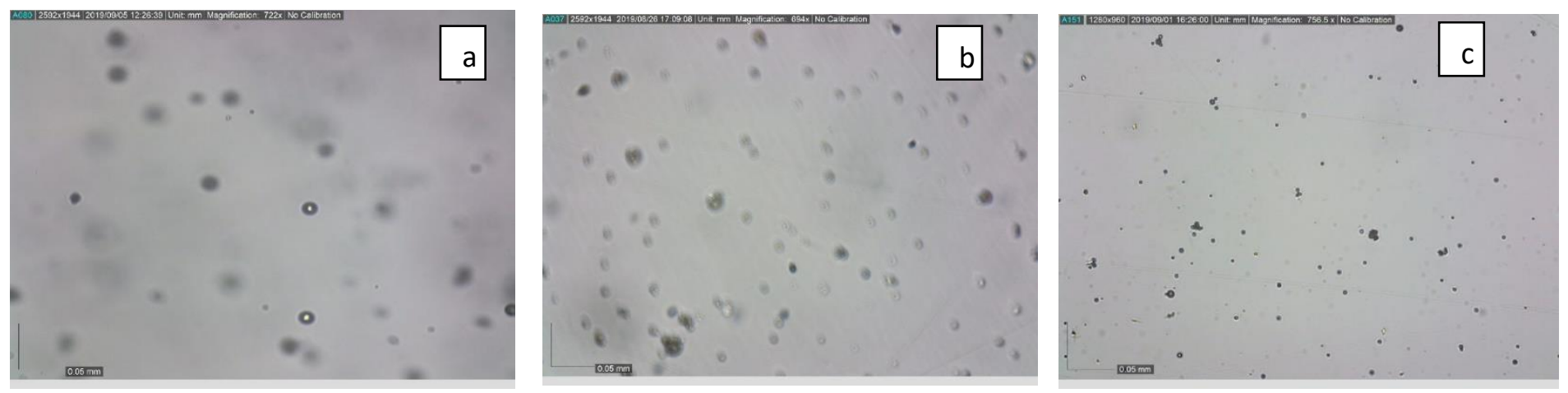

Figure 8: Experiment for polydisperse particles with mean diameter of $10 \mu \mathrm{m}$, sheath flow rate of $1200 \mu \mathrm{L} / \mathrm{min}$ and particle suspension flow rate of $300 \mu \mathrm{L} / \mathrm{min}$, a: particles in outlet reservoir No.1, b: particles in outlet reservoir No.2 and c: particles in outlet reservoir No.3

Table 2: average particle diameter at different outlets in Experiment 2

\begin{tabular}{|cccc|}
\hline $\begin{array}{c}\text { Sheath } \\
\text { flow rate }\end{array}$ & $\begin{array}{c}\text { Particle suspension } \\
\text { flow rate }\end{array}$ & Outlet & $\begin{array}{c}\text { Particles'average } \\
\text { diameter }\end{array}$ \\
\hline & & Outlet 1 & $15.8 \pm 0.02 \mu \mathrm{m}$ \\
$1200 \pm 1 \mu \mathrm{L} / \mathrm{min}$ & $300 \pm 1 \mu \mathrm{L} / \mathrm{min}$ & Outlet 2 & $9.4 \pm 0.02 \mu \mathrm{m}$ \\
& & Outlet 3 & $5.9 \pm 0.02 \mu \mathrm{m}$ \\
\hline
\end{tabular}

For further analysis, microparticle separation with the spiral microchannel, monodisperse polystyrene particles of diameter 5, and $15.6 \mu \mathrm{m}$ were tested, and separation efficiency of microchannel was described. Separation efficiency is defined as the number of one type of particles collected at a specific outlet vs. the total number of the same type of particles collected at all the outlets. Separation efficiency can show the efficiency of a certain type of particles from a whole sample. The flow rate of Sheath flow is $900 \mu \mathrm{L} / \mathrm{min}$, and the flow rate of flow containing particles is 300 $\mu \mathrm{L} / \mathrm{min}$. By imaging the reservoirs embedded in the outputs, as demonstrated in Fig.9, it is observed that particles larger in size have gathered in the first outlet, and smaller particles will exit from the third output.

Moreover, considering that different types of particles will exit from the different outputs in this experiment, the separation efficiency for this state is shown in Fig.10, which illustrates that $15.6 \mu \mathrm{m}$ particle separation efficiency would be about 86 percent and $5 \mu \mathrm{m}$ particles separation efficiency would be about 72 percent. 

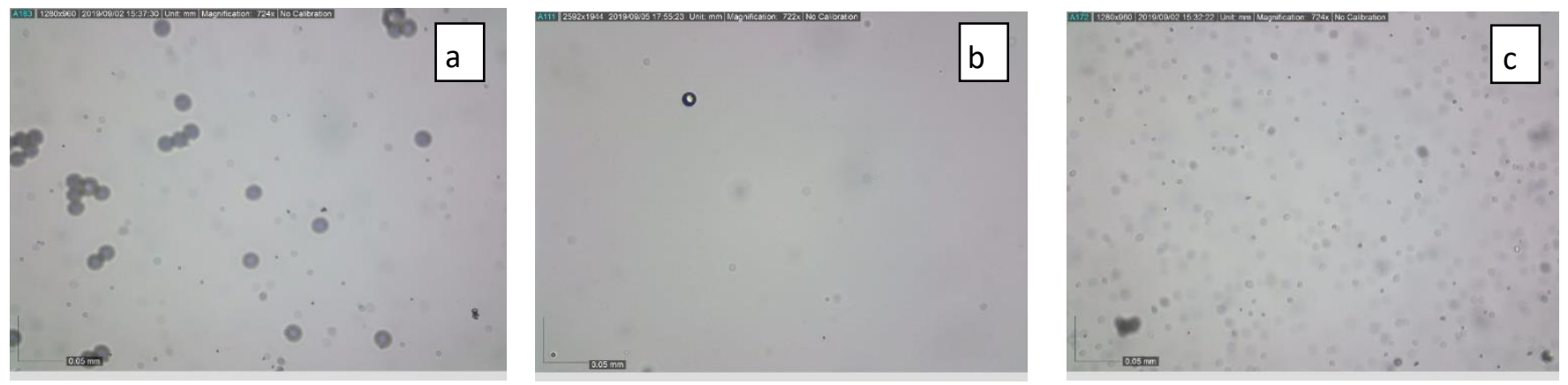

Figure 9: test for monodisperse particles with diameter of $5 \mu \mathrm{m}$ and $15.6 \mu \mathrm{m}$, sheath flow rate of $900 \mu \mathrm{L} / \mathrm{min}$ and particle suspension flow rate of $300 \mu \mathrm{L} / \mathrm{min}$, a: particles in outlet reservoir No.1, b: particles in outlet reservoir No.2 and c: particles in outlet reservoir No.3

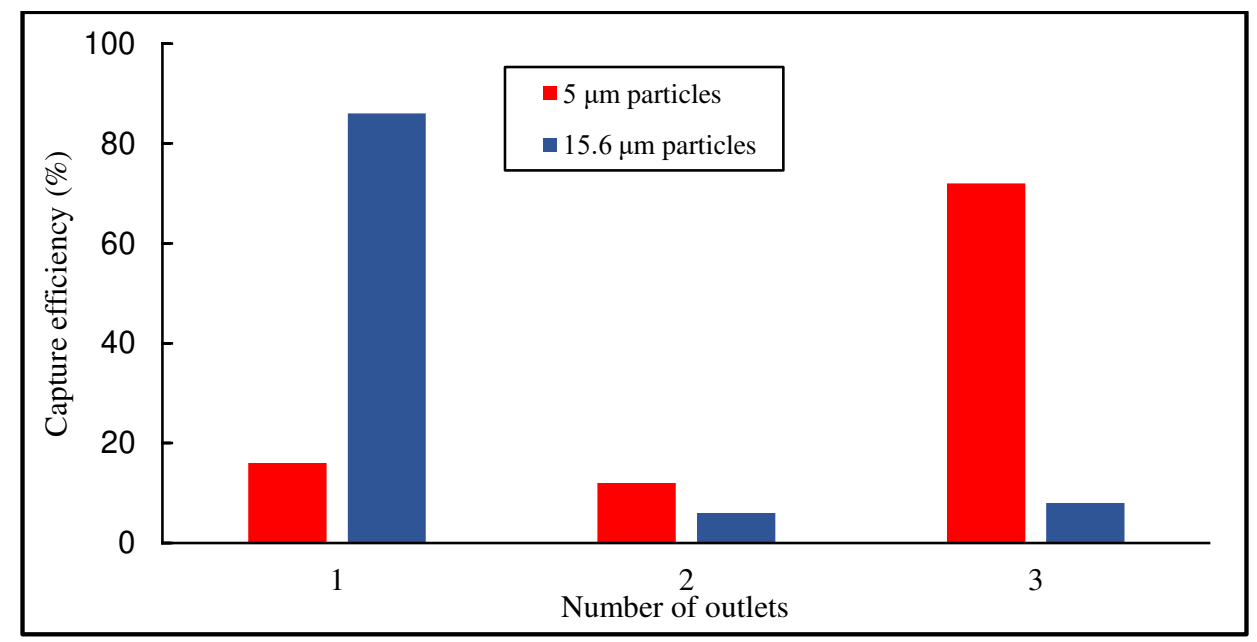

Figure 10: separation efficiency of 5 and $15.6 \mu \mathrm{m}$ particles for each output in flowrate of $900 \mu \mathrm{L} / \mathrm{min}$ for sheath flow

To further analyze, the impact of the sheath buffer flow rate on separation efficiency is investigated. Monodisperse polystyrene particles of diameter 5 and $15.6 \mu \mathrm{m}$ were tested when the flow rate of Sheath flow is $1200 \mu \mathrm{L} / \mathrm{min}$, and the flow rate of particle suspension is $300 \mu \mathrm{L} / \mathrm{min}$. By imaging, the reservoirs embedded in the outputs, separation of microparticles is demonstrated in Fig.11, and consequently, Fig.12 shows the amount of separation efficiency. It can be said that the effect of Dean forces has made the separation efficiency of $5 \mu \mathrm{m}$ particles go up. The separation efficiency for this state is shown in Fig.12, which illustrates $15.6 \mu \mathrm{m}$ particle separation efficiency would be about 84 percent, and $5 \mu \mathrm{m}$ particle separation efficiency would be near 80 percent. 

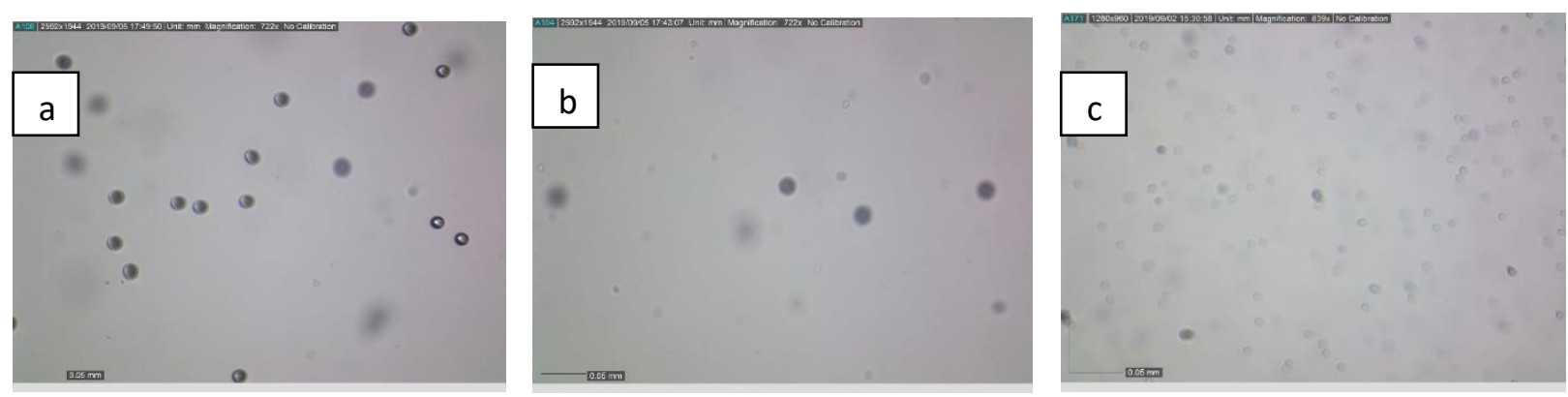

Figure 11: test for monodisperse particles with diameter of $5 \mu \mathrm{m}$ and $15.6 \mu \mathrm{m}$, , sheath flow rate of $1200 \mu \mathrm{L} / \mathrm{min}$ and particle suspension flow rate of $300 \mu \mathrm{L} / \mathrm{min}$, a: particles in outlet reservoir No.1, b: particles in outlet reservoir No.2 and c: particles in outlet reservoir No.3

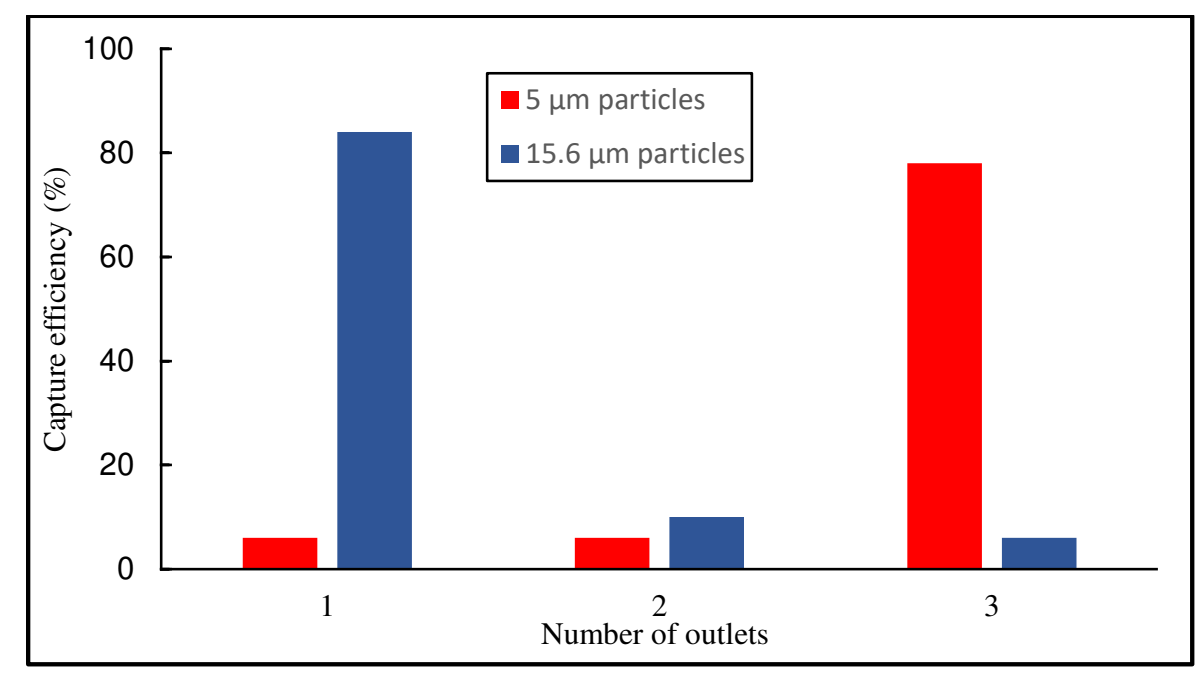

Figure 12: separation efficiency of 5 and $15.6 \mu \mathrm{m}$ particles for each output in flowrate of $900 \mu \mathrm{L} / \mathrm{min}$ for sheath flow.

Since high shear rates could affect cell viability, simulation of laminar flow is performed on the numerical study of fluid shear rate when the flow rate of sheath flow is $1200 \mu \mathrm{L} / \mathrm{min}$. Fig. 13 shows that the amount of shear rate is in a safe area. As shown in Fig.13, the magnitude of shear rate is less than $5 \times 10^{3}(1 / s)$ along almost all the microchannel. 


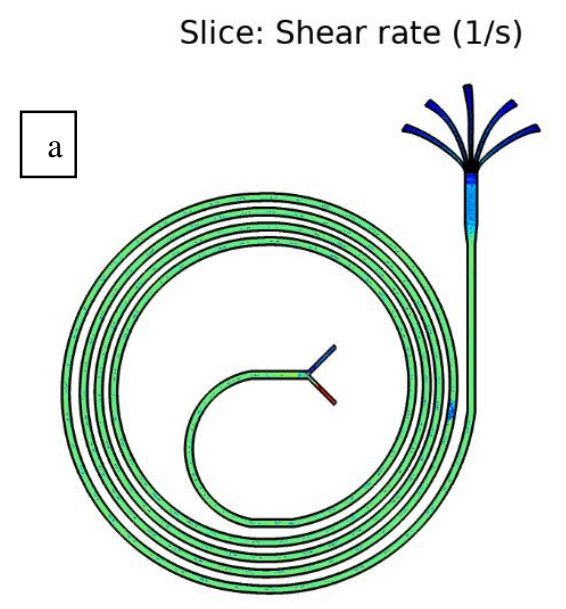

Slice: Velocity magnitude $(\mathrm{m} / \mathrm{s})$

Figure 13: a: shear rate Along the microchannel for mid-section, b: velocity magnitude Along the microchannel for midsection

\section{Conclusion}

We report an inexpensive microfluidic separator with an easy interface based on inertial focusing. For polydisperse HGs particles, at optimal flow conditions, the microchannel collects particles with an average diameter of $15.8 \mu \mathrm{m}$, $9.4 \mu \mathrm{m}$, and $5.9 \mu \mathrm{m}$ at the Intended output. For monodisperse polystyrene particles, the spiral chip achieved an overall separation efficiency of $86 \%$ for $15.6 \mu \mathrm{m}$ particles and about $80 \%$ for $5 \mu \mathrm{m}$ particles. With these outstanding separation performances, this low-cost and user-friendly setup could be used for a variety of microparticle separation applications such as cell separation in biological assays.

\section{Experimental methods}

Microfabrication: The microchannel was fabricated in polydimethylsiloxane (PDMS, Sylgard 184, Dow Corning), using soft lithography techniques. A mixture of PDMS prepolymer and its curing agent (Sylgard 184; Dow Corning, MI) in the ratio of 10:1 was poured on the SU8 2100 (with 2000 RPM) photoresist molds and cured for pre-bake: 5 minute at $\mathrm{T}=65^{\circ} \mathrm{C}$ and 30 minutes at $\mathrm{T}=95^{\circ} \mathrm{C}$; post bake: 5 minute at $65^{\circ} \mathrm{C}$ and 12 minutes at $95^{\circ} \mathrm{C}$; hard bake: 2 minutes at $150^{\circ} \mathrm{C}$. Following curing on a hotplate for 2 hours at $80^{\circ} \mathrm{C}$. Irreversible bonding was made between a PDMS replica and a glass slide, treating both with an oxygen plasma (1.2 -2 mbar, 180 Watt, 2 minutes). Then both PDMS and glass slide was put together and heated at $90^{\circ} \mathrm{C}$ for 15 minutes on a hot plate. The dimensions and angles of the microchannels can be determined by Dinocapture2.0 software from the microscope manufacturer (Fig.14). 

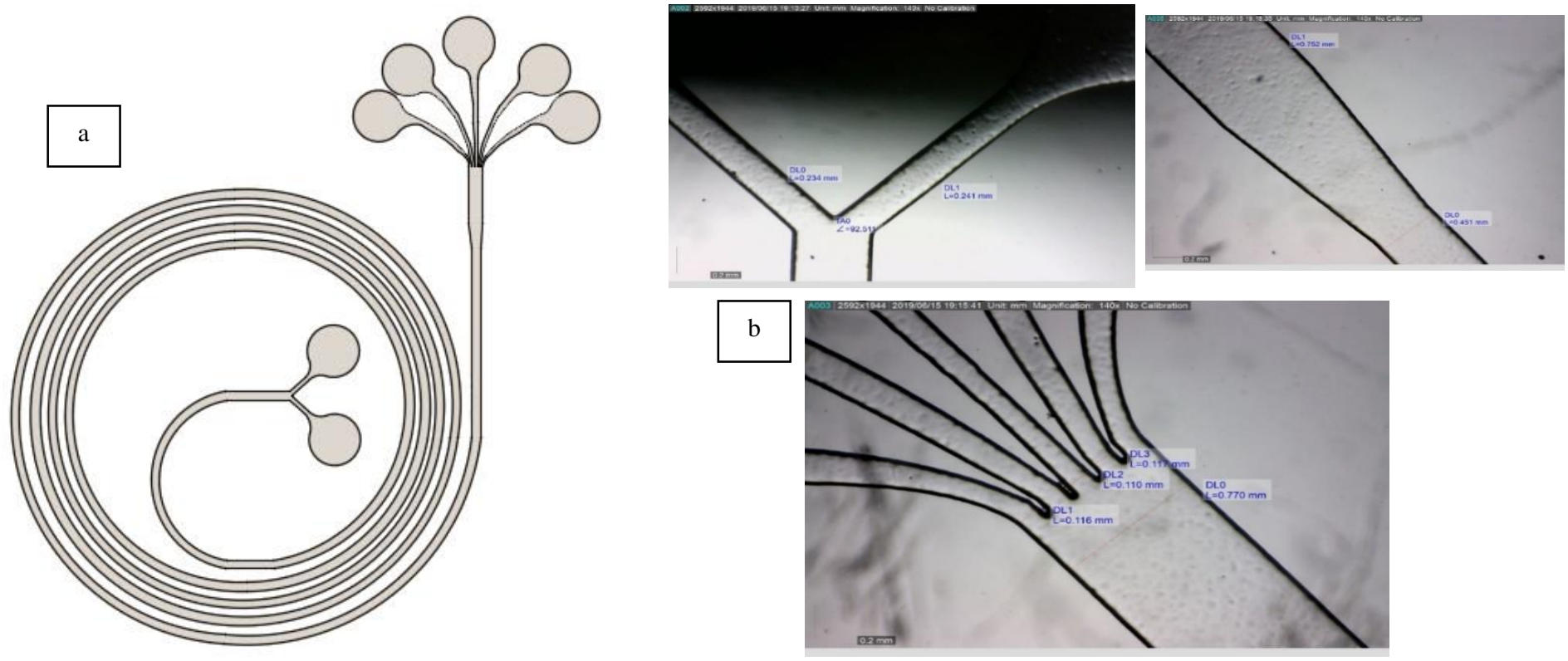

Figure 14: a: The shaped of the desired microchannel and b: various microchannel parts inspected under the microscope

In Diverse experiments, different monodisperse and polydisperse particles which are soluble in pure water are used. Monodisperse particles with diameters of 5 and $15.6 \mu \mathrm{m}$ are made of polystyrene and manufactured by BSPartikel. Polydisperse particles with diameters of 2 to $20 \mu \mathrm{m}$ and an average diameter of $10 \mu \mathrm{m}$ are made of Hallowglass manufactured by Dantec. In order to prevent the effect of the particles on the flow of fluid and other particles, the permitted concentration of the particulate samples should be such that the interaction of the particles in the prepared suspension is minimized. In order to provide a homogeneous suspension with appropriate concentration of the particles, consider the particles in the suspension as shown in Fig.15:

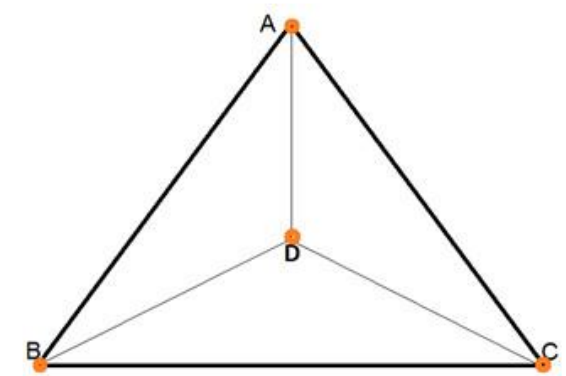

Figure 15: The arrangement of the particles in the suspension is assumed to be at the nodes of a pyramid

Assuming that the particles have the same size and spacing, the geometrical location of the particles in the fluid is assumed to be the prisms of the triangle whose sides are equal to $\mathrm{L}$. The particle spacing should be higher than the 
permissible distance from the simulation studies to avoid interactions between the particles[60]. The volume of each prism is:

$$
V=\frac{\sqrt{2}}{12} L^{3}
$$

Since each particle has four prisms in common and each prism has four vertices, the volume of the constituent of the mixture can be subdivided into prisms. To calculate the numerical concentration of the particles, we have:

$$
n=\frac{N}{V_{\text {Total }}}(12)
$$

Where $\mathrm{N}$ is the total number of particles, and $\mathrm{n}$ is the numerical concentration (number per volume unit). The minimum dimensionless distance must be greater than 10 for the particle to be independent. Therefore, the concentration of the dimensionless dimension can be calculated[60]:

$$
n=\frac{N}{V_{\text {Total }}}=\frac{N}{N \frac{\sqrt{2}}{12} L^{3}}=\frac{12}{\sqrt{2} \chi^{3} D^{3}}
$$

Where $\chi$ is the dimensionless distance for the particles, and it is equal to: $\chi=L / D$. Then, the results of these calculations determine the allowable concentration of particles in the sample fluid containing the particles to avoid the effects of hydrodynamic coupling. In order to ensure one-way coupling between particles and fluid flow, $\chi$ is considered to be 15 in tests.

For imaging and image analysis, the Dino-lite Microscope was used to image the reservoirs embedded in the various microchip outputs, and the recorded images were analyzed using ImageJ1.52a software, powerful software for microscopic image analysis. This software can calculate the distance, cross-section, location, and the number of particles, which is why it is used in the analysis of microscopic images. 


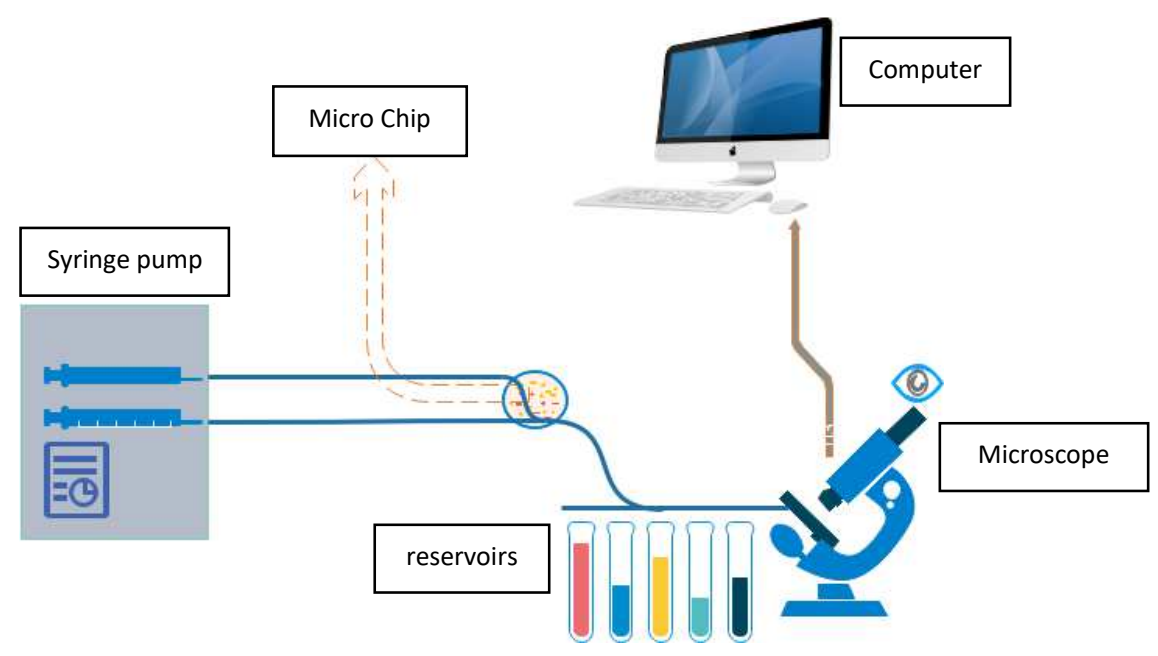

Figure 16: schematic of the separation setup

\section{Decleration}

\section{Acknowledgment}

The authors would like to appreciate and thank the Advanced Measurement Systems Research Laboratory in the mechanical engineering department of Tarbiat Modares University.

\section{Ethics approval and consent to participate}

Not applicable.

\section{Competing interests}

The authors have no competing interests.

\section{Consent for publication}

All authors agreed to submit this study.

\section{Funding}

Not applicable.

\section{Author's contributions}

All the authors have done all the steps together.

\section{Data Availability}

The data that support the findings of this study are available from the corresponding author upon reasonable request. 


\section{References}

[1] A. A. S. Bhagat, H. Bow, H. W. Hou, S. J. Tan, J. Han, and C. T. Lim, "Microfluidics for cell separation," Med. Biol. Eng. Comput., vol. 48, no. 10, pp. 999-1014, 2010, doi: 10.1007/s11517-010-0611-4.

[2] D. R. Gossett et al., "Label-free cell separation and sorting in microfluidic systems," Anal. Bioanal. Chem., vol. 397, no. 8, pp. 3249-3267, Aug. 2010, doi: 10.1007/s00216-010-3721-9.

[3] P. Sajeesh and A. K. Sen, "Particle separation and sorting in microfluidic devices: A review," Microfluid. Nanofluidics, vol. 17, no. 1, pp. 1-52, Nov. 2014, doi: 10.1007/s10404-013-1291-9.

[4] S. Wang et al., "Negative depletion mediated brightfield circulating tumour cell identification strategy on microparticlebased microfluidic chip," J. Nanobiotechnology, vol. 18, no. 1, p. 70, May 2020, doi: 10.1186/s12951-020-00623-4.

[5] K. İçöz et al., "Immunomagnetic separation of B type acute lymphoblastic leukemia cells from bone marrow with flow cytometry validation and microfluidic chip measurements," Sep. Sci. Technol., 2020, doi: 10.1080/01496395.2020.1835983.

[6] A. F. Smith, B. Zhao, M. You, and J. M. Jiménez, "Microfluidic DNA-based potassium nanosensors for improved dialysis treatment," Biomed. Eng. Online, vol. 18, no. 1, p. 73, Jun. 2019, doi: 10.1186/s12938-019-0692-8.

[7] Q. Wu et al., "Organ-on-a-chip: Recent breakthroughs and future prospects," BioMedical Engineering Online, vol. 19, no. 1. BioMed Central Ltd., pp. 1-19, Feb. 12, 2020, doi: 10.1186/s12938-020-0752-0.

[8] D. Di Carlo, "Inertial microfluidics," Lab Chip, vol. 9, no. 21, pp. 3038-3046, 2009, doi: 10.1039/b912547g.

[9] S. S. Kuntaegowdanahalli, A. A. S. Bhagat, G. Kumar, and I. Papautsky, "Inertial microfluidics for continuous particle separation in spiral microchannels.," Lab Chip, vol. 9, no. 20, pp. 2973-2980, Oct. 2009, doi: 10.1039/b908271a.

[10] N. Nivedita and I. Papautsky, "Continuous separation of blood cells in spiral microfluidic devices," Biomicrofluidics, vol. 7, no. 5, p. 054101, 2013, doi: 10.1063/1.4819275.

[11] J. Zhang et al., "Fundamentals and applications of inertial microfluidics: A review," Lab Chip, vol. 16, no. 1, pp. 10-34, 2016, doi: 10.1039/c5lc01159k.

[12] H. Ryu, K. Choi, Y. Qu, T. Kwon, J. S. Lee, and J. Han, "Patient-Derived Airway Secretion Dissociation Technique to Isolate and Concentrate Immune Cells Using Closed-Loop Inertial Microfluidics," Anal. Chem., vol. 89, no. 10, pp. 5549-5556, May 2017, doi: 10.1021/acs.analchem.7b00610.

[13] G. P. Gupta and J. Massagué, “Cancer Metastasis: Building a Framework,” Cell, vol. 127, no. 4, pp. 679-695, Nov. 2006, doi: 10.1016/j.cell.2006.11.001.

[14] T. ASHWORTH, "A case of cancer in which cells similar to those in the tumours were seen in the blood after death," Aust Med J., vol. 14, p. 146, 1869.

[15] K. Ameri et al., "Circulating tumour cells demonstrate an altered response to hypoxia and an aggressive phenotype," $B r$. J. Cancer, vol. 102, no. 3, pp. 561-569, Feb. 2010, doi: 10.1038/sj.bjc.6605491.

[16] E. Lin, T. Cao, S. Nagrath, and M. R. King, "Circulating Tumor Cells: Diagnostic and Therapeutic Applications," Annu. Rev. Biomed. Eng., vol. 20, no. 1, pp. 329-352, 2018, doi: 10.1146/annurev-bioeng-062117-120947.

[17] J. H. Shin, M. G. Lee, S. Choi, and J. K. Park, "Inertia-activated cell sorting of immune-specifically labeled cells in a microfluidic device,” RSC Adv., vol. 4, no. 74, pp. 39140-39144, 2014, doi: 10.1039/c4ra06296e.

[18] A. Y. Fu, C. Spence, A. Scherer, F. H. Arnold, and S. R. Quake, "A microfabricated fluorescence-activated cell sorter," Nat. Biotechnol., vol. 17, no. 11, pp. 1109-1111, Nov. 1999, doi: 10.1038/15095.

[19] J. C. Baret et al., "Fluorescence-activated droplet sorting (FADS): Efficient microfluidic cell sorting based on enzymatic activity," Lab Chip, vol. 9, no. 13, pp. 1850-1858, Jul. 2009, doi: 10.1039/b902504a.

[20] M. M. Wang et al., "Microfluidic sorting of mammalian cells by optical force switching," Nat. Biotechnol., vol. 23, no. 1, pp. 83-87, 2005, doi: 10.1038/nbt1050.

[21] M. Ruzycka, M. R. Cimpan, I. Rios-Mondragon, and I. P. Grudzinski, "Microfluidics for studying metastatic patterns of lung cancer," Journal of Nanobiotechnology, vol. 17, no. 1. BioMed Central Ltd., p. 71, May 27, 2019, doi: 10.1186/s12951-019-0492-0.

[22] J. G. Moreno et al., "Changes in circulating carcinoma cells in patients with metastatic prostate cancer correlate with disease status," Urology, vol. 58, no. 3, pp. 386-392, 2001, doi: 10.1016/S0090-4295(01)01191-8.

[23] H. W. Hou et al., "Isolation and retrieval of circulating tumor cells using centrifugal forces," Sci. Rep., vol. 3, p. 1259, 2013, doi: 10.1038/srep01259.

[24] M. G. Lee, J. H. Shin, C. Y. Bae, S. Choi, and J. Park, "Label-Free Cancer Cell Separation from Human Whole Blood Using Inertial Micro fl uidics at Low Shear Stress," Anal. Chem., vol. 85, no. 13, pp. 6213-6218, 2013.

[25] Q. Chen and J.-M. Lin, Microfluidic Cell Isolation and Recognition for Biomedical Applications. Springer, Singapore., 2018.

[26] W. C. Lee, A. A. S. Bhagat, S. Huang, K. J. Van Vliet, J. Han, and C. T. Lim, "High-throughput cell cycle synchronization using inertial forces in spiral microchannels," Lab Chip, vol. 11, no. 7, p. 1359, 2011, doi: $10.1039 / \mathrm{c} 01 \mathrm{c} 00579 \mathrm{~g}$.

[27] W. Liang et al., "Microfluidic-based cancer cell separation using active and passive mechanisms," Microfluid. Nanofluidics, vol. 24, no. 4, pp. 1-19, Apr. 2020, doi: 10.1007/s10404-020-2331-x.

[28] G. Bai et al., "Characterization of biomechanical properties of cells through dielectrophoresis-based cell stretching and actin cytoskeleton modeling," Biomed. Eng. Online, vol. 16, no. 1, p. 41, Apr. 2017, doi: 10.1186/s12938-017-0329-8.

[29] J. Kim, U. Steinfeld, H. H. Lee, and H. Seidel, "Development of a novel micro immune-magnetophoresis cell sorter," Proc. IEEE Sensors, pp. 1081-1084, 2007, doi: 10.1109/ICSENS.2007.4388593. 
[30] J. D. Adams, U. Kim, and H. T. Soh, "Multitarget magnetic activated cell sorter," Proc. Natl. Acad. Sci., vol. 105, no. 47, pp. 18165-18170, 2008, doi: 10.1073/pnas.0809795105.

[31] M. Zborowski and J. J. Chalmers, "Rare cell separation and analysis by magnetic sorting," Anal. Chem., vol. 83, no. 21, pp. 8050-8056, 2011, doi: 10.1021/ac200550d.

[32] A. Lenshof, C. Magnusson, and T. Laurell, "Acoustofluidics 8: Applications of acoustophoresis in continuous flow microsystems," Lab Chip, vol. 12, no. 7, p. 1210, 2012, doi: 10.1039/c2lc21256k.

[33] A. Manuscript, "Particle separation by dielectrophoresis.pdf," Electrophoresis, vol. 23, no. 13, pp. 1973-1983, 2009, doi: 10.1002/1522-2683(200207)23.

[34] J. Zhang et al., "High-Throughput Separation of White Blood Cells From Whole Blood Using Inertial Microfluidics," IEEE Trans. Biomed. Circuits Syst., vol. In Press, pp. 1-9, 2017, doi: 10.1109/TBCAS.2017.2735440.

[35] M. Pødenphant et al., "Separation of cancer cells from white blood cells by pinched flow fractionation," Lab Chip, vol. 15, no. 24, pp. 4598-4606, 2015, doi: 10.1039/c5lc01014d.

[36] M. Yamada and M. Seki, "Microfluidic particle sorter employing flow splitting and recombining," Anal. Chem., vol. 78, no. 4, pp. 1357-1362, 2006, doi: 10.1021/ac0520083.

[37] H. Tsutsui and C. Ho, "Cell separation by non-inertial force fields in microfluidic systems," Mech. Res. Commun., vol. 36, no. 1, pp. 92-103, 2009, doi: 10.1016/j.mechrescom.2008.08.006.

[38] Z. Wu, B. Willing, J. Bjerketorp, J. K. Jansson, and K. Hjort, "Soft inertial microfluidics for high throughput separation of bacteria from human blood cells," Lab Chip, vol. 9, no. 9, p. 1193, 2009, doi: 10.1039/b817611f.

[39] M. Hosokawa et al., "Size-selective microcavity array for rapid and efficient detection of circulating tumor cells," Anal. Chem., vol. 82, no. 15, pp. 6629-6635, 2010, doi: 10.1021/ac101222x.

[40] Z. Wu, Y. Chen, M. Wang, and A. J. Chung, "Continuous inertial microparticle and blood cell separation in straight channels with local microstructures," Lab Chip, vol. 16, no. 3, pp. 532-542, 2016, doi: 10.1039/C5LC01435B.

[41] G. Segré and A. Silberberg, "Radial particle displacements in poiseuille flow of suspensions," Nature, vol. 189, no. 4760, pp. 209-210, 1961, doi: 10.1038/189209a0.

[42] M. E. Warkiani et al., "Slanted spiral microfluidics for the ultra-fast, label-free isolation of circulating tumor cells," Lab Chip, vol. 14, no. 1, pp. 128-137, Jan. 2014, doi: 10.1039/c31c50617g.

[43] M. E. brahim. Warkiani et al., "Ultra-fast, label-free isolation of circulating tumor cells from blood using spiral microfluidics," Nat. Protoc., vol. 11, no. 1, pp. 134-148, 2016, doi: 10.1038/nprot.2016.003.

[44] P. Y. Yeh et al., "An Efficient Spiral Microchannel for Continuous Small Particle Separations," Sensors Actuators B. Chem., 2017, doi: 10.1016/j.snb.2017.06.037.

[45] M. S. Syed et al., "Selective separation of microalgae cells using inertial microfluidics," Bioresour. Technol., vol. 252, no. December 2017, pp. 91-99, Mar. 2018, doi: 10.1016/j.biortech.2017.12.065.

[46] J. H. Lee, S. K. Lee, J. H. Kim, and J. H. Park, "Separation of particles with bacterial size range using the control of sheath flow ratio in spiral microfluidic channel," Sensors Actuators, A Phys., vol. 286, pp. 211-219, 2019, doi: 10.1016/j.sna.2018.12.047.

[47] N. M. Karabacak et al., "Microfluidic, marker-free isolation of circulating tumor cells from blood samples," Nat. Protoc., vol. 9, no. 3, pp. 694-710, 2014, doi: 10.1038/nprot.2014.044.

[48] A. Abdulla, W. Liu, A. Gholamipour-Shirazi, J. Sun, and X. Ding, "High-Throughput Isolation of Circulating Tumor Cells Using Cascaded Inertial Focusing Microfluidic Channel,” Anal. Chem., vol. 90, no. 7, pp. 4397-4405, Apr. 2018, doi: 10.1021/acs.analchem.7b04210.

[49] M. G. Lee, J. H. Shin, S. Choi, and J. K. Park, "Enhanced blood plasma separation by modulation of inertial lift force," Sensors Actuators, B Chem., vol. 190, pp. 311-317, 2014, doi: 10.1016/j.snb.2013.08.092.

[50] S. O. Catarino, R. O. Rodrigues, D. Pinho, J. M. Miranda, G. Minas, and R. Lima, "Blood Cells Separation and Sorting Techniques of Passive Microfluidic Devices: From Fabrication to Applications," Micromachines, vol. 10, no. 9, p. 593, Sep. 2019, doi: 10.3390/mi10090593.

[51] E. Grassilli, “A Microfluidic Device for Label-Free Sorting of Circulating Tumor Cells," University of Illinois at Chicago, 2018.

[52] Y. Zhou, Z. Ma, and Y. Ai, "Sheathless inertial cell focusing and sorting with serial reverse wavy channel structures," Microsystems Nanoeng., vol. 4, no. 1, pp. 1-4, Dec. 2018, doi: 10.1038/s41378-018-0005-6.

[53] D. H. Yoon, J. B. Ha, Y. K. Bahk, T. Arakawa, S. Shoji, and J. S. Go, "Size-selective separation of micro beads by utilizing secondary flow in a curved rectangular microchannel," Lab Chip, vol. 9, no. 1, pp. 87-90, 2009, doi: 10.1039/b809123d.

[54] W. R. Dean, “ LXXII. The stream-line motion of fluid in a curved pipe (Second paper),” London, Edinburgh, Dublin Philos. Mag. J. Sci., vol. 5, no. 30, pp. 673-695, Apr. 1928, doi: 10.1080/14786440408564513.

[55] N. Nivedita, P. Ligrani, and I. Papautsky, "Dean Flow Dynamics in Low-Aspect Ratio Spiral Microchannels," Sci. Rep., vol. 7, no. October 2016, pp. 1-10, 2017, doi: 10.1038/srep44072.

[56] T. Kwon, R. Yao, J. F. P. Hamel, and J. Han, "Continuous removal of small nonviable suspended mammalian cells and debris from bioreactors using inertial microfluidics," Lab Chip, vol. 18, no. 18, pp. 2826-2837, 2018, doi: 10.1039/c8lc00250a.

[57] M. Hejazian, W. Li, and N. T. Nguyen, "Lab on a chip for continuous-flow magnetic cell separation," Lab Chip, vol. 15, no. 4, pp. 959-970, 2015, doi: 10.1039/c4lc01422g.

[58] D. Pappas, "Microfluidics and cancer analysis: Cell separation, cell/tissue culture, cell mechanics, and integrated 
analysis systems," Analyst, vol. 141, no. 2, pp. 525-535, 2016, doi: 10.1039/c5an01778e.

[59] Z. H. Fan, Circulating tumor cells : isolation and analysis. .

[60] M. Zabetian, M. S. Saidi, M. B. Shafii, and M. H. Saidi, "Separation of microparticles suspended in a minichannel using laser radiation pressure," Appl. Opt., vol. 52, no. 20, p. 4950, 2013, doi: 10.1364/ao.52.004950. 
Figures
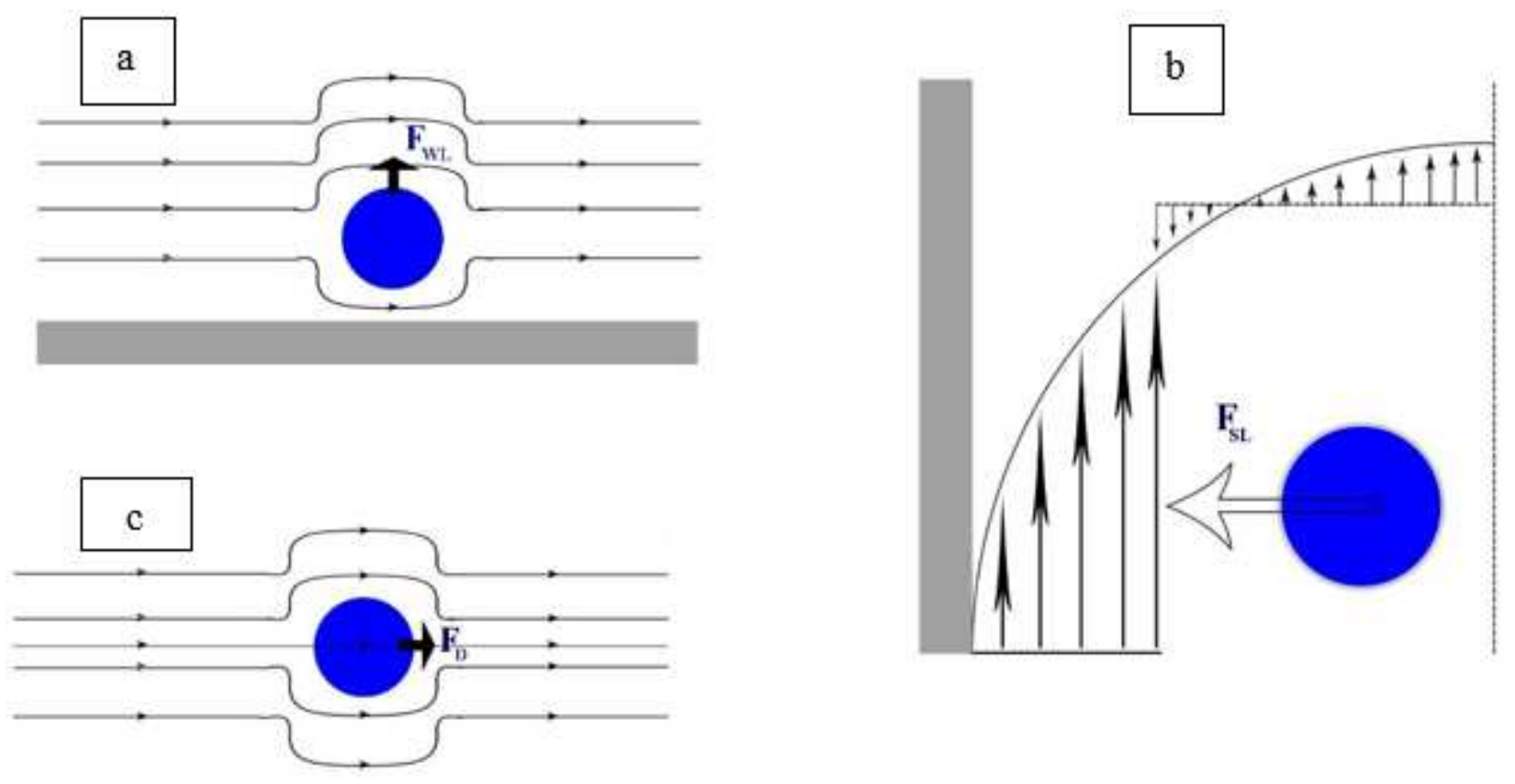

Figure 1

a: Wall-induced lift force that causes the particle to move away from the walls due to the effect of the wall and to approach the center of the channel, b: Shear-induced lift force caused by the parabolic flow velocity profile of the fluid and directs the particle to the sides of the channel, c: Viscous drag force along the axis of flow [25]
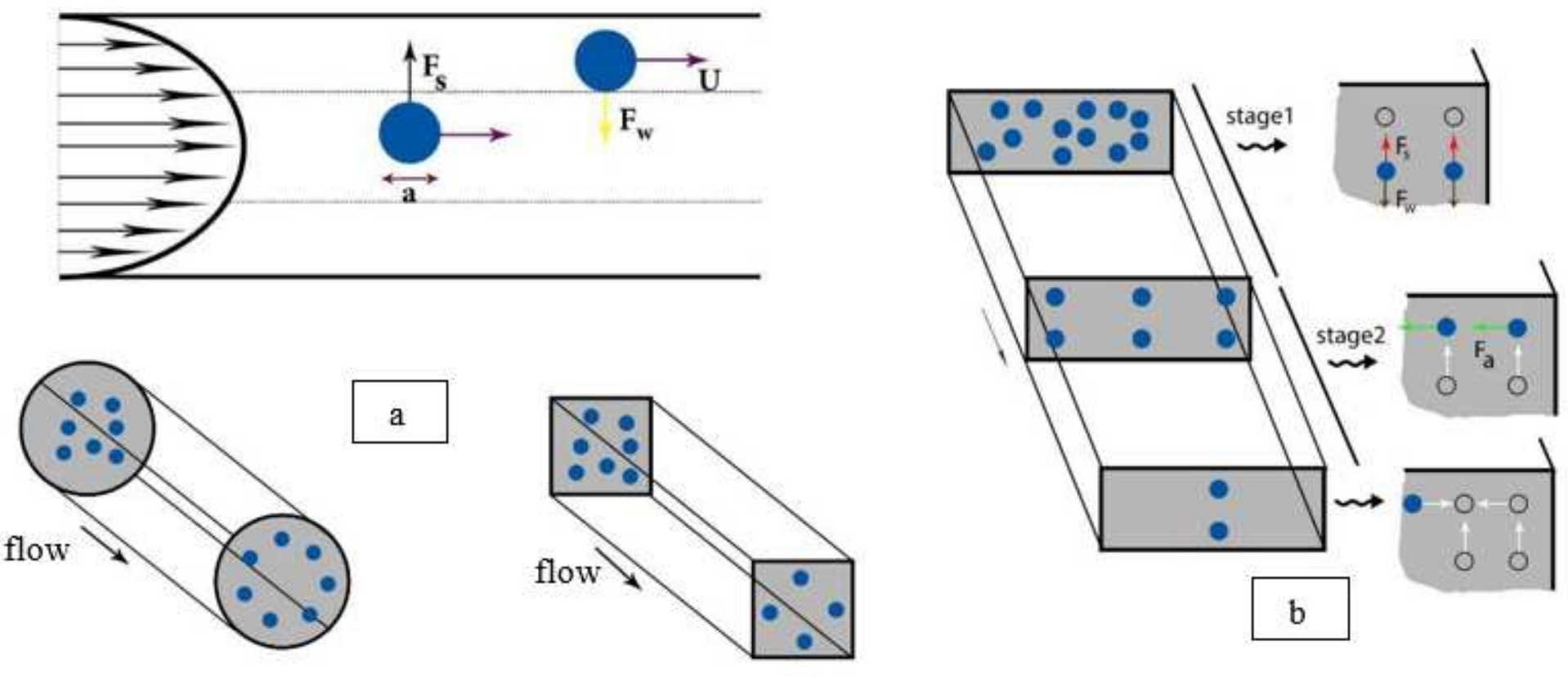

Figure 2 
Equilibrium positions of particles in microchannels with different cross-sectional area indicate that a: in circular and square microchannels, the number of positions is high and, b: by reducing the aspect ratios, it is possible to reduce the interchangeable positions similar to rectangular cross-section[10]

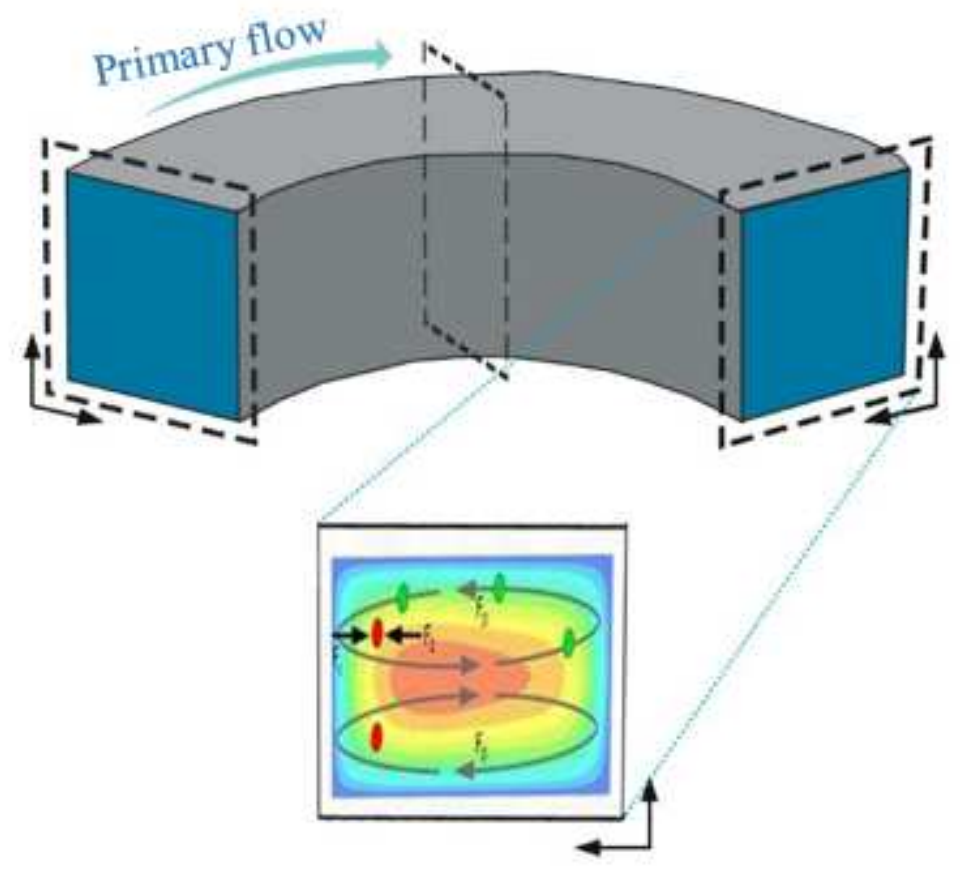

Figure 3

The Dean flow. In curved channels, when inertia is of the highest importance, the faster movement of liquids near the center of the channel tends to continue externally, and in order to maintain mass, more stagnant fluid circulates inwardly near the walls. These two anti-rotation vortices create a perpendicular motion to the mainstream direction[8].

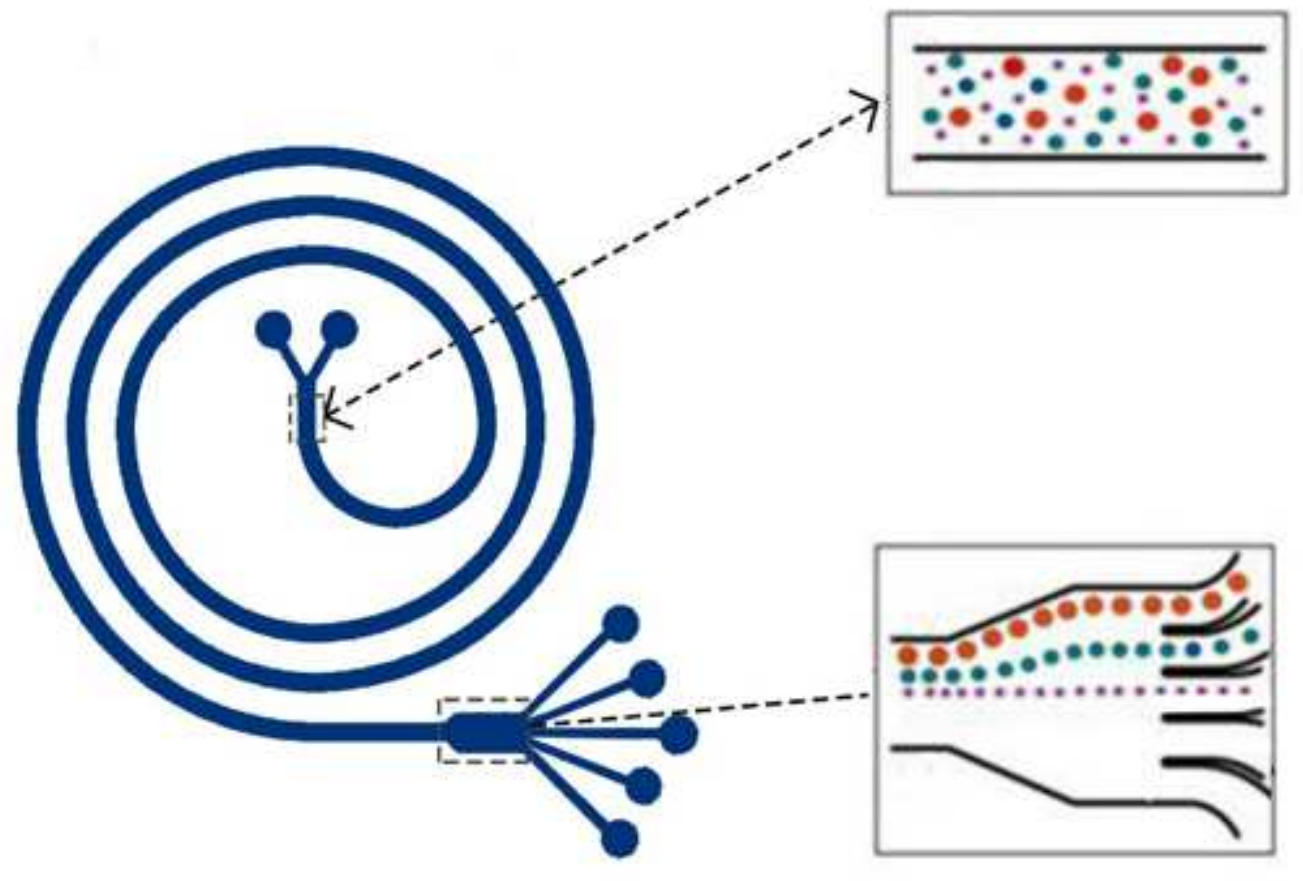

Figure 4 
Schematic of the spiral microparticle separator. The randomly dispersed particles equilibrate at different equilibrium positions along the inner wall (IW) of the spiral microchannel under the influence of FL and FD. The separation between individual particle streams is enhanced by connecting the spiral section into a diffuser section, before conducting the individual streams using multiple outlet ports[9].

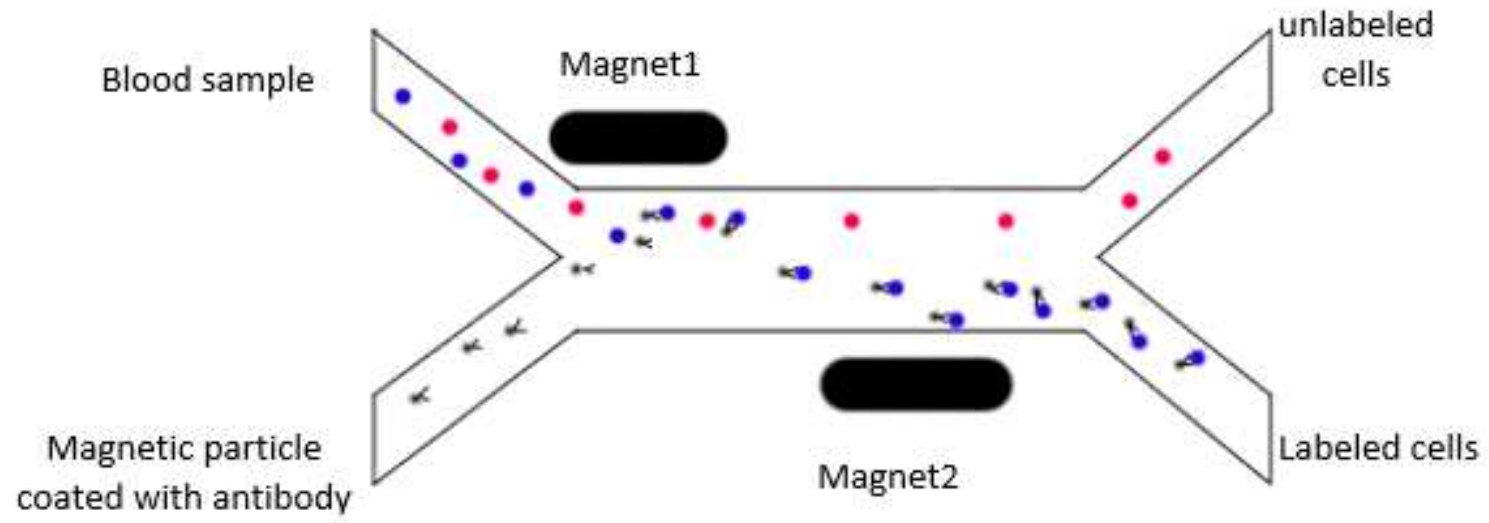

Figure 5

In magnetic microfluidic separation, the arbitrary cells are labeled by antibodies and are pulled out of the flow path via applied magnetic force.

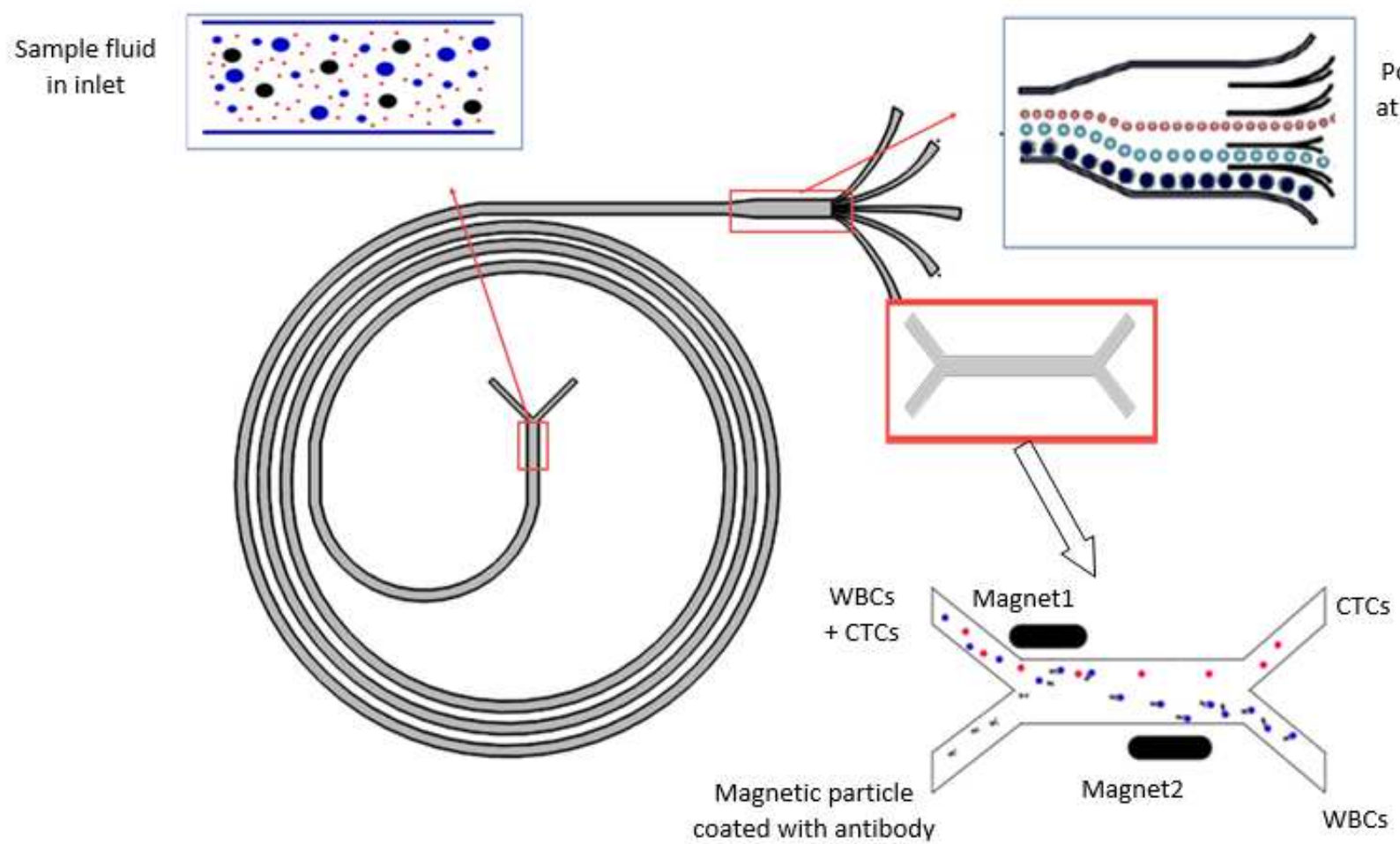

\section{Figure 6}

Schematic of the designed separator system: in the first part of the microchannel, the particles are separated by inertia and size-based technique. Both cancer cells and magnetic-labeled particles have 
been removed. In the magnetic separation section of the labeled white blood cells, they deflect from the current path-line, and as a result, the circulating cancer cells will be selected negatively.
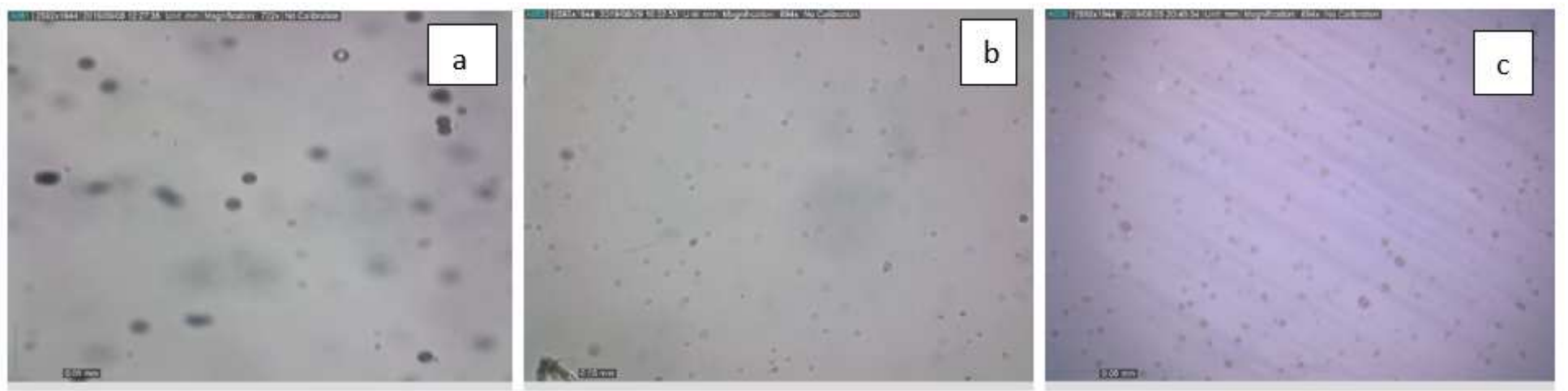

\section{Figure 7}

Experiment for polydisperse particles with mean diameter of $10 \mu \mathrm{m}$, sheath flow rate of $900 \mu \mathrm{L} / \mathrm{min}$ and particle suspension flow rate of $300 \mu \mathrm{L} / \mathrm{min}$, a: particles in outlet reservoir No.1, b: particles in outlet reservoir No.2 and c: particles in outlet reservoir No.3
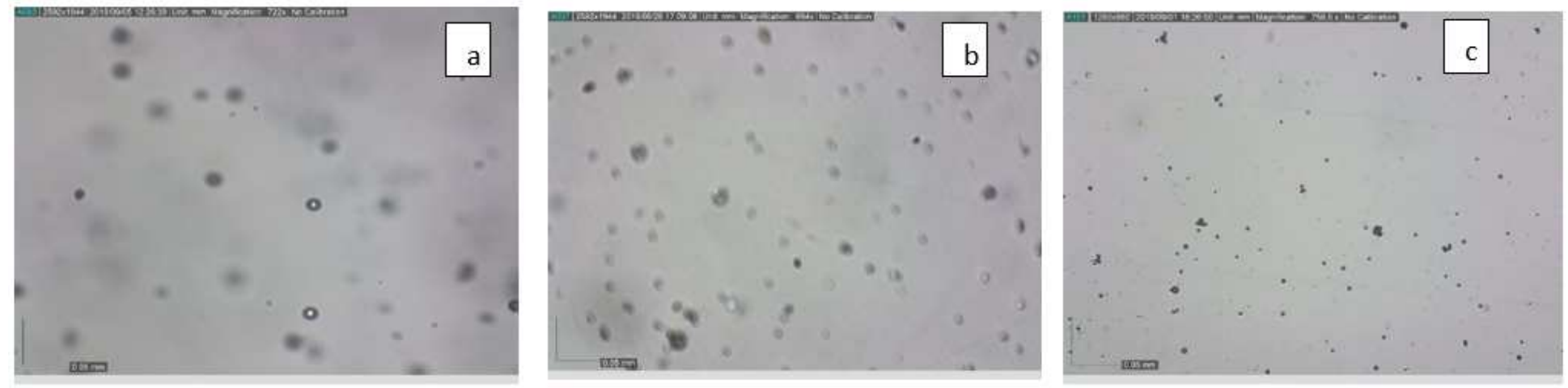

\section{Figure 8}

Experiment for polydisperse particles with mean diameter of $10 \mu \mathrm{m}$, sheath flow rate of $1200 \mu \mathrm{L} / \mathrm{min}$ and particle suspension flow rate of $300 \mu \mathrm{L} / \mathrm{min}$, a: particles in outlet reservoir No.1, b: particles in outlet reservoir No.2 and c: particles in outlet reservoir No.3
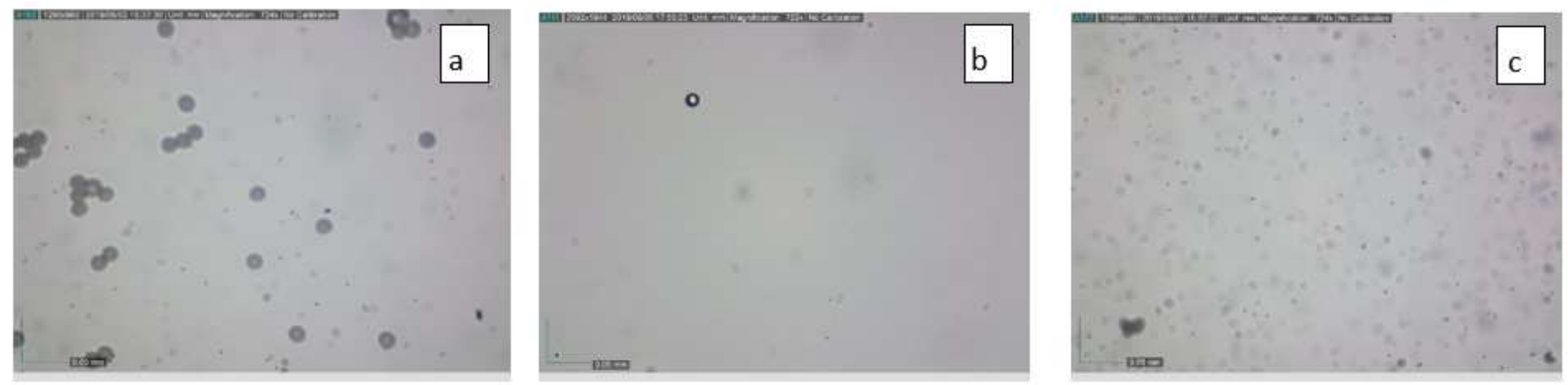

\section{Figure 9}


test for monodisperse particles with diameter of $5 \mu \mathrm{m}$ and $15.6 \mu \mathrm{m}$, sheath flow rate of $900 \mu \mathrm{L} / \mathrm{min}$ and particle suspension flow rate of $300 \mu \mathrm{L} / \mathrm{min}$, a: particles in outlet reservoir No.1, b: particles in outlet reservoir No.2 and c: particles in outlet reservoir No.3

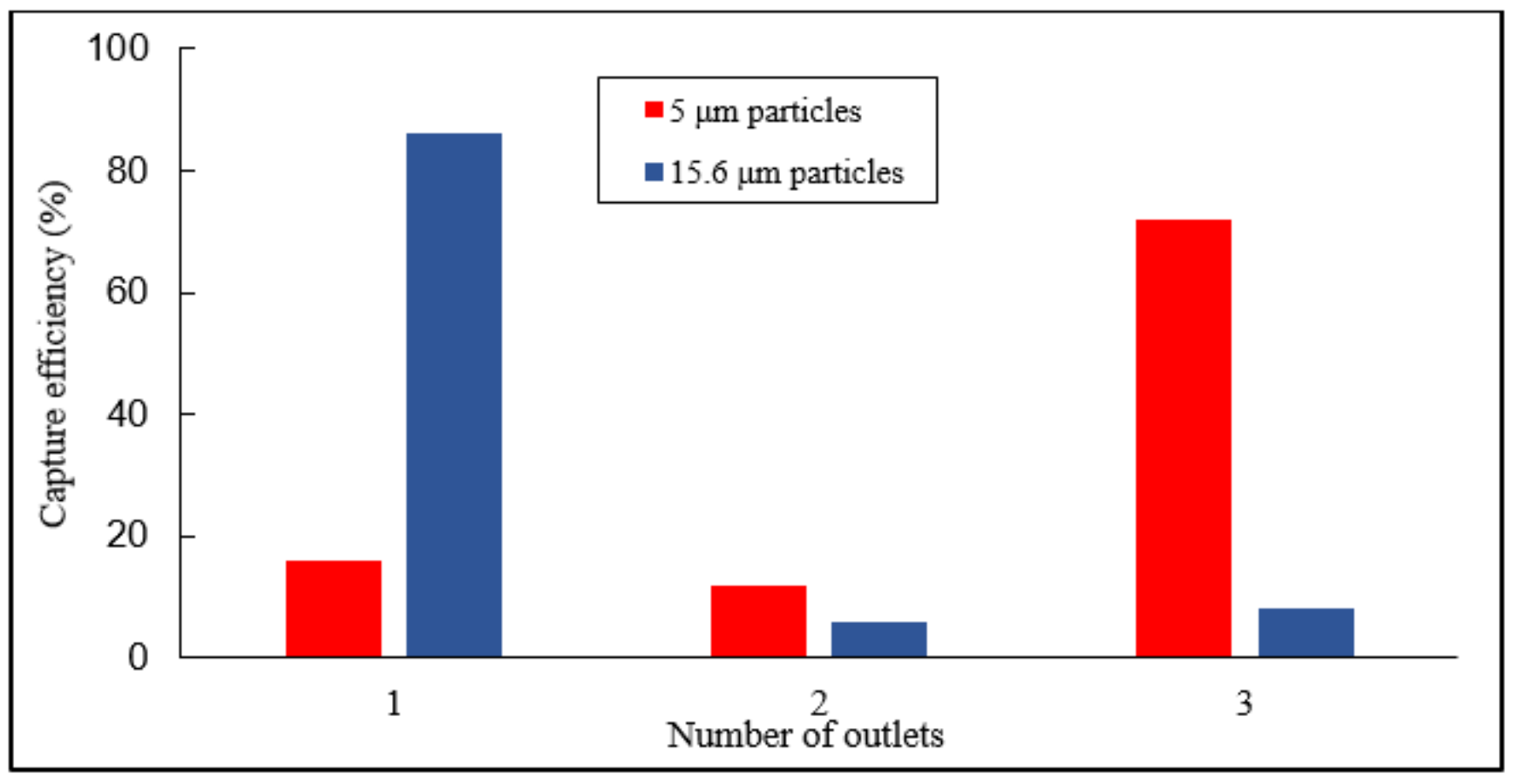

Figure 10

separation efficiency of 5 and $15.6 \mu \mathrm{m}$ particles for each output in flowrate of $900 \mu \mathrm{L} / \mathrm{min}$ for sheath flow
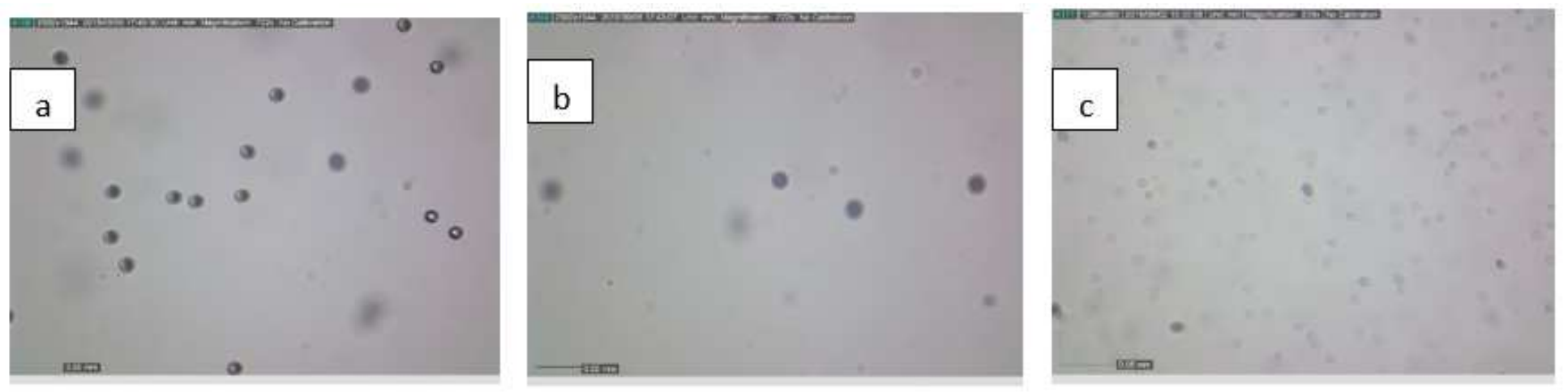

\section{Figure 11}

test for monodisperse particles with diameter of $5 \mu \mathrm{m}$ and $15.6 \mu \mathrm{m}$, , sheath flow rate of $1200 \mu \mathrm{L} / \mathrm{min}$ and particle suspension flow rate of $300 \mu \mathrm{L} / \mathrm{min}$, a: particles in outlet reservoir No.1, b: particles in outlet reservoir No. 2 and c: particles in outlet reservoir No.3 


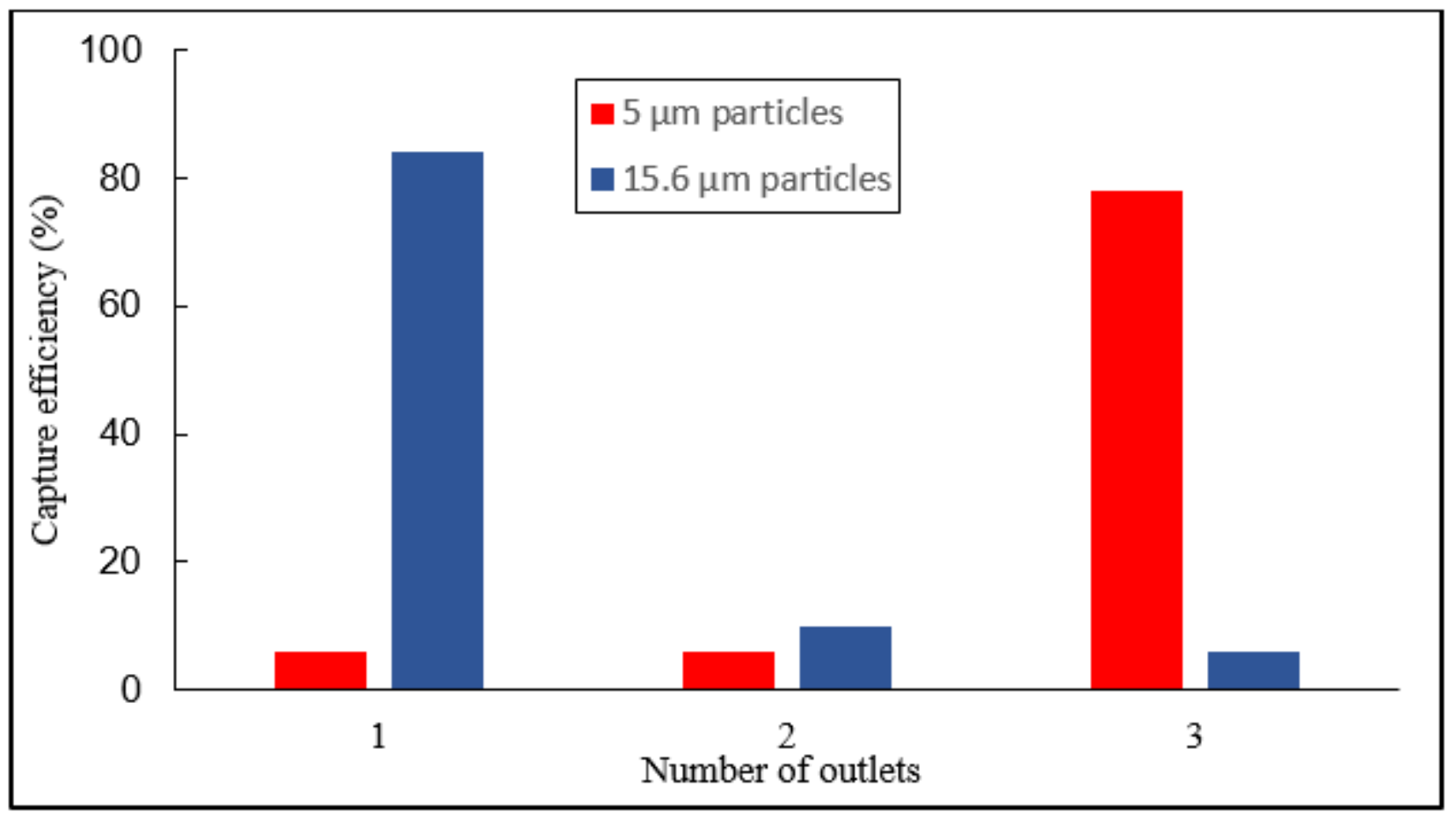

Figure 12

separation efficiency of 5 and $15.6 \mu \mathrm{m}$ particles for each output in flowrate of $900 \mu \mathrm{L} / \mathrm{min}$ for sheath flow.

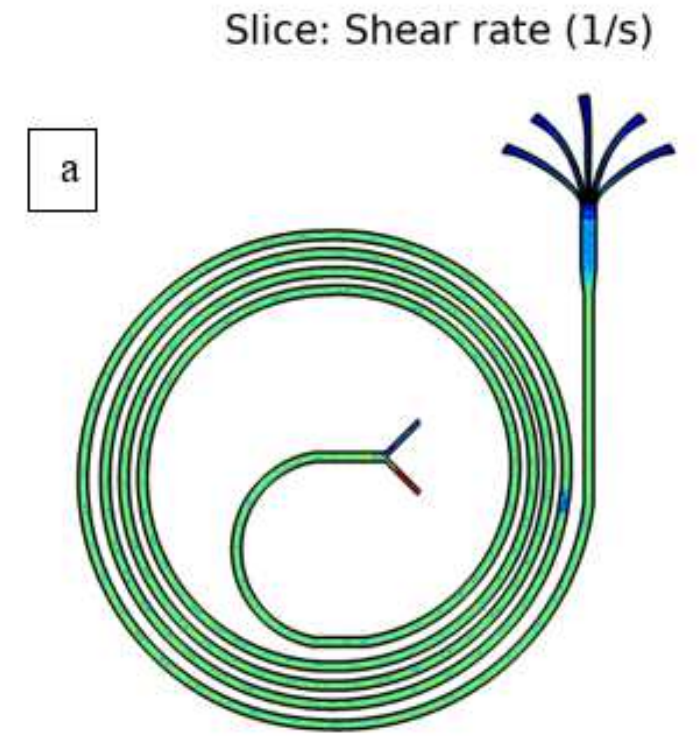

Slice: Velocity magnitude $(\mathrm{m} / \mathrm{s})$
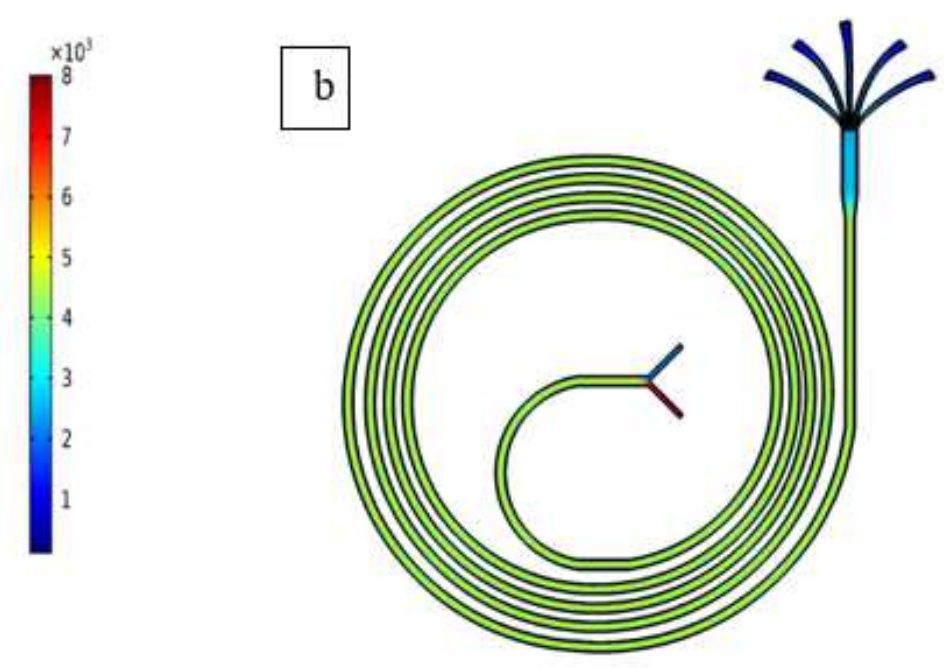

Figure 13

a: shear rate Along the microchannel for mid-section, b: velocity magnitude Along the microchannel for mid-section 

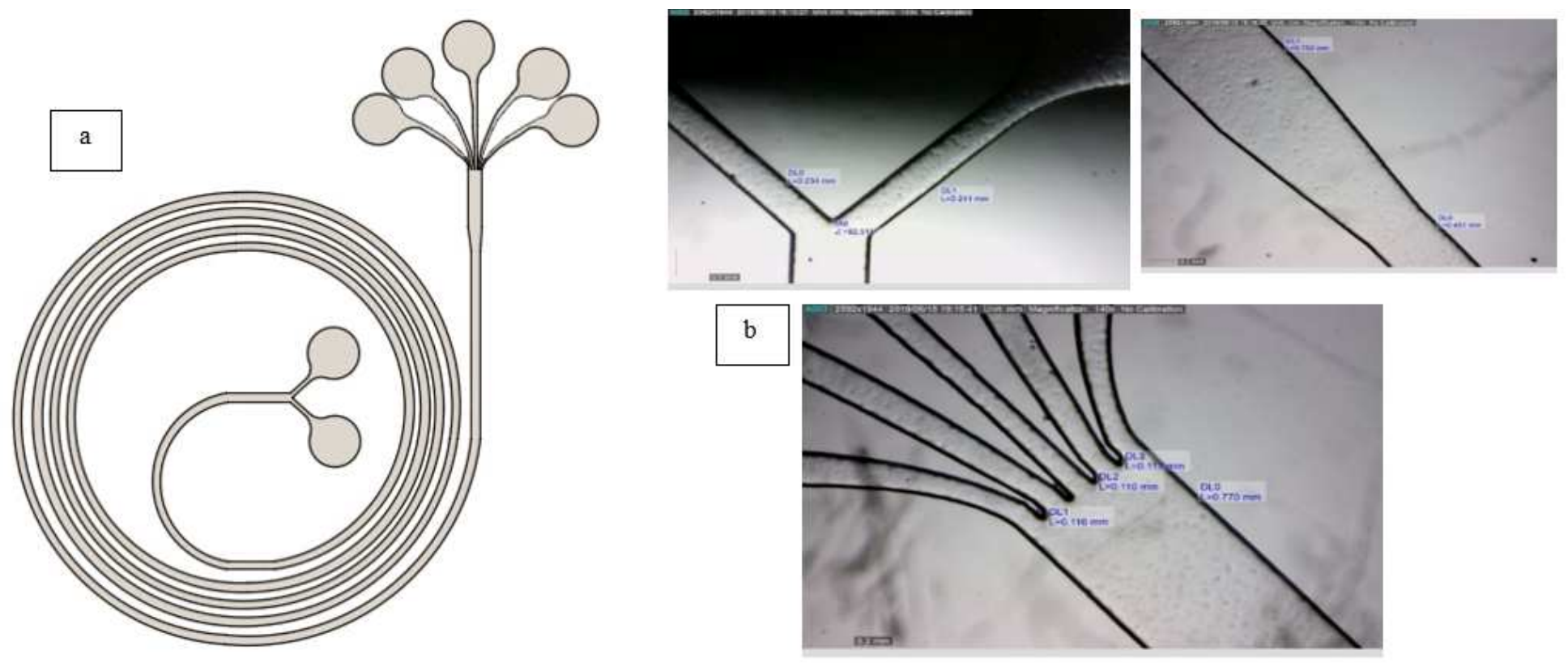

Figure 14

a: The shaped of the desired microchannel and b: various microchannel parts inspected under the microscope

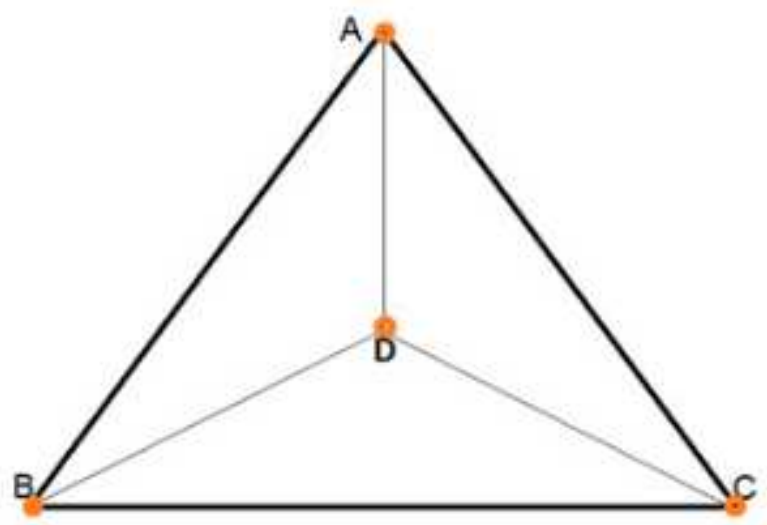

Figure 15

The arrangement of the particles in the suspension is assumed to be at the nodes of a pyramid 


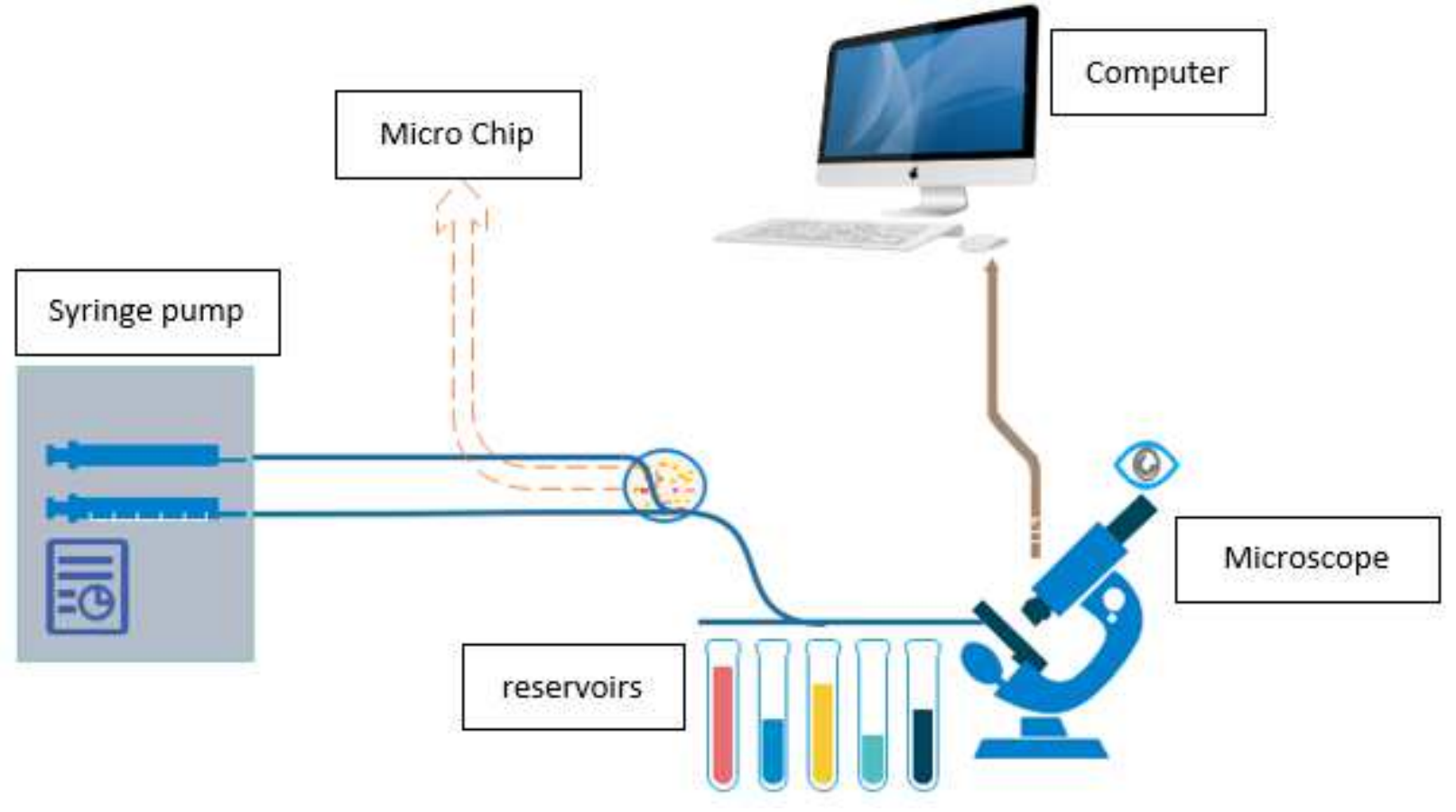

Figure 16

schematic of the separation setup 